\title{
The Effects of High Glucose on Adipogenic and Osteogenic Differentiation of Gestational Tissue-Derived MSCs
}

\author{
Weerawan Hankamolsiri, ${ }^{1}$ Sirikul Manochantr, ${ }^{2,3}$ Chairat Tantrawatpan,,3 \\ Duangrat Tantikanlayaporn, ${ }^{2,3}$ Pairath Tapanadechopone, ${ }^{4}$ and Pakpoom Kheolamai ${ }^{2,3}$
}

${ }^{1}$ Medical Sciences Program (Cellular and Molecular Biology), Faculty of Medicine, Thammasat University, Pathumthani 12120, Thailand

${ }^{2}$ Division of Cell Biology, Faculty of Medicine, Thammasat University, Pathumthani 12120, Thailand

${ }^{3}$ Center of Excellence in Stem Cell Research, Thammasat University, Pathumthani 12120, Thailand

${ }^{4}$ Department of Psychiatry, Faculty of Medicine, Thammasat University, Pathumthani 12120, Thailand

Correspondence should be addressed to Pakpoom Kheolamai; pkheolamai@me.com

Received 23 June 2015; Revised 31 August 2015; Accepted 15 September 2015

Academic Editor: Laura Lasagni

Copyright (C) 2016 Weerawan Hankamolsiri et al. This is an open access article distributed under the Creative Commons Attribution License, which permits unrestricted use, distribution, and reproduction in any medium, provided the original work is properly cited.

\begin{abstract}
Most type 2 diabetic patients are obese who have increased number of visceral adipocytes. Those visceral adipocytes release several factors that enhance insulin resistance making diabetic treatment ineffective. It is known that significant percentages of visceral adipocytes are derived from mesenchymal stem cells and high glucose enhances adipogenic differentiation of mouse bone marrow-derived MSCs (BM-MSCs). However, the effect of high glucose on adipogenic differentiation of human bone marrow and gestational tissue-derived MSCs is still poorly characterized. This study aims to investigate the effects of high glucose on proliferation as well as adipogenic and osteogenic differentiation of human MSCs derived from bone marrow and several gestational tissues including chorion, placenta, and umbilical cord. We found that high glucose reduced proliferation but enhanced adipogenic differentiation of all MSCs examined. The expression levels of some adipogenic genes were also upregulated when MSCs were cultured in high glucose. Although high glucose transiently downregulated the expression levels of some osteogenic genes examined, its effect on the osteogenic differentiation levels of the MSCs is not clearly demonstrated. The knowledge gained from this study will increase our understanding about the effect of high glucose on adipogenic differentiation of MSCs and might lead to an improvement in the diabetic treatment in the future.
\end{abstract}

\section{Introduction}

Diabetes mellitus (DM) is a disease in which blood sugar level of patients is abnormally high. Diabetic patients are at risk of developing several disabling and life-threatening complications including cardiovascular diseases, cerebrovascular diseases, diabetic nephropathy, and diabetic retinopathy [13]. The important contributing factor for diabetic development is obesity which is defined as an excessive accumulation of body fat. Adipocytes in the accumulated fat tissues, especially around waist area and visceral organs, release several cytokines which reduce the sensitivity of several cell types to insulin leading to insulin resistant and type 2 diabetes $[4,5]$.

It is known that adipocytes are derived, at least in part, from multipotent mesenchymal stem cells (MSCs). Several recent studies demonstrated that high glucose reduced proliferation of rat bone marrow-derived MSCs (BM-MSCs) $[6,7]$ but enhanced adipogenic differentiation and lipid accumulation of mouse BM-MSCs [8], human osteosarcoma cell MG63 $[9,10]$ and human muscle-derived stem cells [11]. An increase in adipogenic differentiation levels was accompanied by an upregulation of the expression levels of several adipogenic genes including peroxisome proliferatoractivated receptor gamma $(P P A R \gamma)$, adipocyte protein 2 $(A P 2)$, adipose tissue-specific secretory factor $(A D S F)$, sterol regulatory element-binding protein $1 \mathrm{C}$ (SREBP1C), lipoprotein lipase $(L P L)$, adiponectin $(A D I P O Q)$, and glucose transporter type 4 (GLUT4) $[8,9,11]$. In contrast to adipogenic differentiation, high glucose suppressed osteogenic differentiation and downregulated the expression levels of osteogenic 
gene runt-related transcription factor 2 (RUNX2), collagen type I (COL1A1), osteonectin $(O N)$, and osteocalcin $(O C N)$ of mouse BM-MSCs and MG63 cells [7, 9].

Although the effects of high glucose on the proliferation and differentiation of rat and mouse BM-MSCs have been previously reported, the effects of high glucose on biological properties of human MSCs derived from gestational tissues which, due to their ease of isolation by noninvasive procedure, are considered more suitable sources of MSCs for clinical applications have yet to be determined. Therefore, the present study aims to investigate the effects of high glucose on proliferation and adipogenic and osteogenic differentiation of MSCs derived from bone marrow and several gestational tissues including chorion, placenta, and umbilical cord, as well as the mechanisms underlying those effects.

\section{Materials and Methods}

2.1. Subjects. This study was approved by the Institutional Review Board, Faculty of Medicine, Thammasat University, which was in accordance with the Declaration of Helsinki, the Belmont Report, CIOMS Guidelines, and ICH-GCP. Human bone marrow samples were obtained from healthy volunteers after giving written informed consents. The gestational tissues (umbilical cord, placenta, and chorion) were obtained from healthy newborns after receiving written informed consents from their mothers.

2.2. Isolation and Culture of MSCs. Mononuclear cells (MNCs) from human bone marrow were isolated using Ficoll-Hypaque (GE Healthcare, USA) density gradient centrifugation $\left(2,000 \mathrm{rpm}\right.$ for 30 minutes at $\left.20^{\circ} \mathrm{C}\right)$. Bone marrowderived MNCs were then cultured in complete medium (Dulbecco's Modified Eagle's Medium (DMEM; Gibco BRL, USA) supplemented with $10 \%$ (v/v) fetal bovine serum (FBS; Gibco BRL, USA), 2 mM L-glutamine (Gibco BRL, USA), $100 \mathrm{U} / \mathrm{mL}$ penicillin, and $100 \mu \mathrm{g} / \mathrm{mL}$ streptomycin (Gibco BRL, USA)) at the density of $1 \times 10^{6}$ cells $/ \mathrm{cm}^{2}$. Cultures were maintained at $37^{\circ} \mathrm{C}$ in a humidified atmosphere containing $5 \% \mathrm{CO}_{2}$ and medium was replaced every 3 days throughout the entire culture period.

For the isolation of MSCs from umbilical cord, placenta, and chorion, the tissues were minced into small pieces and digested by incubating with $1.6 \mathrm{mg} / \mathrm{mL}$ collagenase XI (Sigma-Aldrich, USA) and $200 \mathrm{mg} / \mathrm{mL}$ deoxyribonuclease I (Sigma-Aldrich, USA) for 4 hours at $37^{\circ} \mathrm{C}$ with shaking. After incubation, the cells were washed twice with phosphate buffered saline (PBS; Gibco BRL, USA), resuspended in complete medium, and plated into $25 \mathrm{~cm}^{2}$ tissue culture flask (Corning, USA). Cultures were maintained at $37^{\circ} \mathrm{C}$ in a humidified atmosphere containing $5 \% \mathrm{CO}_{2}$ and medium was replaced every 3 days throughout the entire culture period.

2.3. Immunophenotyping of MSCs. The 3rd-5th passage MSCs were characterized for their surface marker expressions by being incubated with the following mouse antihuman antibodies: anti-CD45-FITC (BioLegend, USA), antiCD34-PE (BioLegend, USA), anti-CD90-FITC (BioLegend,
USA), anti-CD73-PE (BioLegend, USA), and anti-CD105$\mathrm{PE}$ (BD Pharmingen, USA) for 30 minutes at $4^{\circ} \mathrm{C}$ in the dark. After incubation, cell pellets were washed twice with PBS and fixed with $1 \%(\mathrm{w} / \mathrm{v})$ paraformaldehyde in PBS. Flow cytometry was performed by FACScalibur flow cytometer (Becton Dickinson, USA) using CellQuest software (Becton Dickinson, USA).

2.4. Osteogenic and Adipogenic Differentiation of MSCs. The 3rd-5th passage MSCs were used to assess their adipogenic and osteogenic differentiation potential. For adipogenic differentiation, $7.5 \times 10^{4}$ cells were seeded into an individual well of 6-well plate (Corning, USA) and cultured in adipogenic differentiation medium (complete medium supplemented with $0.1 \mu \mathrm{M}$ dexamethasone (Sigma-Aldrich, USA), $1 \mu \mathrm{M}$ insulin (Sigma-Aldrich, USA), $200 \mu \mathrm{M}$ indomethacin (Sigma-Aldrich, USA), and $250 \mu \mathrm{M}$ isobutylmethylxanthine (Sigma-Aldrich, USA)). Cultures were maintained at $37^{\circ} \mathrm{C}$ in a humidified atmosphere containing $5 \% \mathrm{CO}_{2}$ and medium was replaced every 3 days throughout the entire culture period. After culture for 3 weeks, cells were fixed with formalin vapor, stained with $0.5 \%(\mathrm{w} / \mathrm{v})$ oil red O (Sigma-Aldrich, USA) in isopropanol for 20 minutes at room temperature, and observed under light microscope (Nikon TS100, Japan) to determine number of MSC-derived adipocytes.

For osteogenic differentiation, $3 \times 10^{4}$ cells were seeded into an individual well of 6 -well plate (Corning, USA) and cultured in osteogenic differentiation medium (complete medium supplemented with $0.1 \mu \mathrm{M}$ dexamethasone (SigmaAldrich, USA), $50 \mu \mathrm{g} / \mathrm{mL}$ L-ascorbic acid (Sigma-Aldrich, USA), and $10 \mathrm{mM} \beta$-glycerophosphate (Sigma-Aldrich, USA)). Cultures were maintained at $37^{\circ} \mathrm{C}$ in a humidified atmosphere containing $5 \% \mathrm{CO}_{2}$ and medium was replaced every 3 days throughout the entire culture period. After culture for 3 weeks, cells were fixed with $4 \%$ paraformaldehyde and subjected to alkaline phosphatase activity assay using BCIP/NBT liquid substrate (Sigma-Aldrich, USA) to determine levels of osteogenic differentiation.

\subsection{Effects of High Glucose on the Functional Properties of MSCs}

2.5.1. Effect on Proliferation. $1 \times 10^{3} 3 \mathrm{rd}-5$ th passage MSCs were seeded into an individual well of 24 -well plate (Corning, USA) containing $500 \mu \mathrm{L}$ complete medium supplemented with $25 \mathrm{mM}$ D-glucose which have been shown to be an optimal concentration of D-glucose for simulating high glucose condition by several previous studies $[7-9,11]$. Cultures were maintained at $37^{\circ} \mathrm{C}$ in a humidified atmosphere containing $5 \% \mathrm{CO}_{2}$ and medium was replaced every 3 days throughout the entire culture period. Cells were harvested on culture days $2,4,6,8,10,12$, and 14 to determine cell number. Dead cells were excluded by Trypan blue staining (Sigma-Aldrich, USA) and the number of viable cells was determined by hemocytometer. The average number of cells at each time point was then calculated and plotted against culture time to show growth kinetic. MSCs cultured in complete medium without D-glucose supplementation serve as controls. 
2.5.2. Effect on Adipogenic Differentiation. $7.5 \times 10^{4} 3 \mathrm{rd}-5$ th passage MSCs were seeded into an individual well of 6-well plates (Corning, USA) containing adipogenic differentiation medium supplemented with $25 \mathrm{mM} \mathrm{D}$-glucose. Cultures were maintained at $37^{\circ} \mathrm{C}$ in a humidified atmosphere containing $5 \% \mathrm{CO}_{2}$ and medium was replaced every 3 days throughout the entire culture period. The number of adipocyte-like cells was determined on culture day 14 (for BM-MSCs) and day 28 (for CH-MSCs, PL-MSCs, and UC-MSCs) by oil red $\mathrm{O}$ staining. To measure the amount of oil red $\mathrm{O}$ staining in each sample, oil red $\mathrm{O}$ presented in each stained sample was extracted by incubation with $1 \mathrm{~mL}$ isopropanol for 5 minutes at room temperature. The optical density (OD) of each extracted oil red $\mathrm{O}$ sample was measured by microplate reader (BioTex, USA) at the wavelength of $500 \mathrm{~nm}$. The concentration of extracted oil red $\mathrm{O}$ in each sample was then calculated by comparing the measured OD values with a standard curve generated from various known concentrations of oil red O. MSCs cultured in adipogenic differentiation medium without $\mathrm{D}$-glucose supplementation serve as controls.

2.5.3. Effect on Osteogenic Differentiation. $3 \times 10^{4} 3 \mathrm{rd}-5$ th passage MSCs were seeded into an individual well of 6-well plates (Corning, USA) containing osteogenic differentiation medium supplemented with $25 \mathrm{mM}$ D-glucose. Cultures were maintained at $37^{\circ} \mathrm{C}$ in a humidified atmosphere containing $5 \% \mathrm{CO}_{2}$ and medium was replaced every 3 days throughout the entire culture period. After 14-day culture period, the levels of osteogenic differentiation were determined by alkaline phosphatase activity assay using BCIP/NBT liquid substrate (Sigma-Aldrich, USA) according to the manufacturer's instruction. MSCs cultured in osteogenic differentiation medium without $\mathrm{D}$-glucose supplementation serve as controls.

2.5.4. Effects on the Expression Levels of Adipogenic and Osteogenic Genes. To determine the effects of high glucose on the expression levels of adipogenic genes, $2 \times 10^{5} 3 \mathrm{rd}-$ 5th passage MSCs were cultured in $25 \mathrm{~cm}^{2}$ tissue culture flasks (Corning, USA) containing adipogenic differentiation medium supplemented with $25 \mathrm{mM}$ D-glucose. On culture day 14 (for BM-MSCs) and culture day 28 (for CH-MSCs, PL-MSCs, and UC-MSCs), the expression levels of five adipogenic genes including ADIPOQ, GLUT4, LPL, PPAR $\gamma$, and $S R E B P 1 C$ were determined by quantitative real-time PCR (qRT-PCR). MSCs cultured in adipogenic differentiation medium without D-glucose supplementation serve as controls.

To determine the effect of high glucose on the expression levels of osteogenic genes, $2 \times 10^{5} 3$ rd-5th passage MSCs were cultured in $25 \mathrm{~cm}^{2}$ tissue culture flasks containing osteogenic differentiation medium supplemented with $25 \mathrm{mM}$ D-glucose. On culture days 7 and 14, the expression levels of three osteogenic genes including RUNX2, OSX, and OCN were determined by qRT-PCR. MSCs cultured in osteogenic differentiation medium without D-glucose supplementation serve as controls.
2.6. Quantitative Real-Time PCR ( $q R T-P C R)$. Total RNAs were isolated from cells using PureLink RNA Mini Kit (Invitrogen Corporation, USA) according to the manufacturer's instruction. cDNA was then synthesized from $2 \mu \mathrm{g}$ of RNA using SuperScript III Reverse Transcriptase (Invitrogen Corporation, USA). MicroAmp fast optical 96well reaction plate (Applied Biosystem, USA) was used for qRT-PCR. Each well contained $3 \mu \mathrm{L}$ of cDNA, $1 \mu \mathrm{L}$ of $10 \mu \mathrm{M}$ forward and reverse primer mix, and $10 \mu \mathrm{L}$ of SYBR Green PCR Master Mix (Applied Biosystem, USA). Plates were sealed with MicroAmp clear adhesive film (Applied Biosystem, USA) to prevent evaporation of the reactant. PCR was performed using StepOne plus real-time PCR system (Applied Biosystem, USA) using the following protocol: $95^{\circ} \mathrm{C}$ initial denaturation for 10 minutes, followed by 40 cycles of denaturation $\left(95^{\circ} \mathrm{C}, 10\right.$ seconds), annealing $\left(60^{\circ} \mathrm{C}, 10\right.$ seconds), and extension $\left(72^{\circ} \mathrm{C}, 40\right.$ seconds). Each sample was examined in duplicate and mean value was calculated. The quantitation was based on normalizing the level of gene of interest to the invariant control gene glyceraldehyde 3phosphate dehydrogenase (GAPDH). Data were analyzed by comparative CT method using StepOne Software version 2.2 (Applied Biosystems; ABI, USA) and presented as relative mRNA levels. The primer sequences were listed in Table 1.

2.7. Statistical Analysis. Data were presented as mean \pm standard error of the mean (SEM). Paired Student's $t$-tests were used to assess the significance of differences between observed data. $P<0.05$ was considered to be statistically significant.

\section{Result}

3.1. Characteristics of Bone Marrow and Gestational TissueDerived MSCs. MSCs derived from gestational tissues including placenta (PL-MSCs), umbilical cord (UC-MSCs), and chorion (CH-MSCs) exhibited similar characteristics to that of bone marrow-derived MSCs (BM-MSCs). The gestational tissue-derived MSCs displayed fibroblast-like morphology (Figure 1(a)), expressed typical MSC surface markers (positive for CD73, CD90, CD105, and negative for hematopoietic markers CD34 and CD45; Figure 1(b)), and could differentiate to adipocytes and osteocytes as demonstrated by oil red $\mathrm{O}$ staining (Figure 1(c)) and alkaline phosphatase activity assay (Figure $1(\mathrm{~d})$ ), respectively. It is worth noting that CH-MSC, PL-MSCs, and UC-MSCs took a longer period of time to differentiate to adipocytes compared with BM-MSCs (28 days versus 14 days). Furthermore, the numbers of adipocyte-like cells generated from $\mathrm{CH}$ MSC, PL-MSCs, and UC-MSCs were also lower than those generated from BM-MSCs.

3.2. Effect of High Glucose on MSC Proliferation. To study the effect of high glucose on MSC proliferation, BM-MSCs, $\mathrm{CH}-$ MSCs, UC-MSCs, and PL-MSCs were cultured in complete medium supplemented with $25 \mathrm{mM} \mathrm{D}$-glucose for 14 days. The results showed that, from day 6 onward, the proliferation of BM-MSCs cultured in high glucose condition was significantly reduced when comparing with their normal glucose 
TABLE 1: The sequences of primers for qRT-PCR.

\begin{tabular}{lcc}
\hline Gene & \multicolumn{1}{c}{ Forward primer } & \multicolumn{1}{c}{ Reverse primer } \\
\hline ADIPOQ & $5^{\prime}$-CCTGGTGAGAAGGGTGAGAA-3' & $5^{\prime}$-CAATCCCACACTGAATGCTG-3' \\
GLUT4 & $5^{\prime}$-CTTCGAGACAGCAGGGGTAG-3' & $5^{\prime}$-ACAGTCATCAGGATGGCACA-3' \\
LPL & $5^{\prime}$-TCAACTGGATGGAGGAGGAG-3' & $5^{\prime}$-GGGGCTTCTGCATACTCAAA-3' \\
PPARY & $5^{\prime}$-GACCACTCCCACTCCTTTGA-3' & $5^{\prime}$-AGGCTCCACTTTGATTGCAC-3' \\
SREBP1C & $5^{\prime}$-TTCTCACCTCCCAGCTCTGT-3' & $5^{\prime}$-GGAGGCTTCTTTGCTGTGAG-3 \\
RUNX2 & $5^{\prime}$-GACAGCCCCAACTTCCTGT-3' \\
OCN & $5^{\prime}$-CTCACACTCCTCGCCCTATT-3' & $5^{\prime}$-CCGGAGCTCAGCAGAATAAT-3' \\
OSX & $5^{\prime}$-TGCTTGAGGAGGAAGTTCAC-3' & $5^{\prime}$-TCAGCCAACTCGTCACAGTC-3' \\
\hline
\end{tabular}

ADIPOQ: adiponectin, GLUT4: glucose transporter type 4, LPL: lipoprotein lipase, PPAR $\gamma$ : peroxisome proliferator-activated receptor gamma, SREBP1C: sterol regulatory element-binding protein 1C, RUNX2: runt-related transcription factor 2, OCN: osteocalcin, OSX: osterix, and GAPDH: glyceraldehyde 3-phosphate dehydrogenase.

controls (Figure 2). Similar to BM-MSCs, the proliferation of CH-MSCs, PL-MSCs, and UC-MSCs cultured in high glucose condition was also significantly reduced when comparing with their normal glucose controls (Figure 2).

3.3. Effect of High Glucose on Adipogenic Differentiation of MSCs. To study the effect of high glucose on adipogenic differentiation of BM-MSCs, BM-MSCs were cultured in adipogenic differentiation medium supplemented with $25 \mathrm{mM}$ D-glucose for 14 days. At the end of culture, the cells were stained with oil red $\mathrm{O}$ to determine number of adipocytelike cells (Figure 3(a)). The results showed that the number of adipocyte-like cells and the percentages of adipocyte-like cells in total cell number derived from BM-MSCs cultured in high glucose condition were significantly greater than those derived from their normal glucose controls $(1308.7 \pm 28.8$ versus $879.0 \pm 75.7,{ }^{*} P<0.05$, and $10.1 \pm 0.6$ versus $5.0 \pm 1.3$, ${ }^{*} P<0.05$, resp.) (Figures $3(\mathrm{~b})$ and $3(\mathrm{c})$ ). In agreement with these results, the concentration of oil red $\mathrm{O}$ extracted from BM-MSCs cultured in high glucose after oil red $\mathrm{O}$ staining was also significantly greater than that of their normal glucose controls $\left(271.2 \pm 17.5\right.$ versus $\left.199.4 \pm 18.0,{ }^{*} P<0.05\right)$ (Figure 3(d)). In addition to the number of adipocyte-like cells, the mRNA levels of adipogenic genes PPAR $\gamma$ and $L P L$ of BM-MSCs cultured in high glucose condition were also significantly upregulated when comparing with their normal glucose controls (Figure 3(e)). In contrast to $P P A R \gamma$ and $L P L$, the mRNA levels of other adipogenic genes including SREBP1C, ADIPOQ, and GLUT4 of BM-MSCs cultured in high glucose were not different from their normal glucose controls (Figure 3(e)).

Similar to BM-MSCs, the numbers of adipocyte-like cells derived from CH-MSCs, PL-MSCs, and UC-MSCs cultured in high glucose condition were significantly greater than those derived from their normal glucose controls [751.7 \pm 17.6 versus $639.0 \pm 30.2,{ }^{*} P<0.05$ for $\mathrm{CH}-\mathrm{MSCs} ; 919.0 \pm 18.9$ versus 707.3 $\pm 42.8,{ }^{*} P<0.05$ for PL-MSCs; and 803.3 \pm 23.3 versus $698.3 \pm 11.7,{ }^{*} P<0.05$ for UC-MSCs] (Figures 4 (a) and $4(\mathrm{~b}))$. The percentages of adipocyte-like cells in total cell number derived from CH-MSCs, PL-MSCs, and UC-MSCs cultured in high glucose condition were also significantly greater than those derived from their normal glucose controls $\left[5.7 \pm 0.3\right.$ versus $3.9 \pm 0.4,{ }^{*} P<0.05$ for $\mathrm{CH}-\mathrm{MSCs} ; 17.1 \pm 2.3$ versus $8.8 \pm 0.9,{ }^{*} P<0.05$ for PL-MSCs; and $6.7 \pm 0.5$ versus $3.9 \pm 0.2,{ }^{*} P<0.05$ for UC-MSCs] (Figure 4(c)). In agreement with these results, the concentrations of oil red $\mathrm{O}$ extracted from CH-MSCs, PL-MSCs, and UC-MSCs cultured in high glucose condition after oil red $\mathrm{O}$ staining were also significantly greater than those of their normal glucose controls $\left[537.1 \pm 20.0\right.$ versus $431.5 \pm 23.6,{ }^{*} P<0.05$ for $\mathrm{CH}$ MSCs; $510.7 \pm 15.6$ versus $463.2 \pm 4.0,{ }^{*} P<0.05$ for PLMSCs; and 251.1 \pm 14.8 versus $189.4 \pm 8.4,{ }^{*} P<0.05$ for UCMSCs] (Figure 4(d)). In addition to the number of adipocytelike cells, the mRNA levels of adipogenic genes ADIPOQ and $L P L$ of CH-MSCs and UC-MSCs cultured in high glucose condition were also upregulated when comparing with their normal glucose controls (Figure 4(e)). In contrast to $A D I P O Q$ and $L P L$, the mRNA levels of other adipogenic genes including PPAR $\gamma, S R E B P 1 C$, and GLUT4 in CH-MSCs and UC-MSCs cultured in high glucose were not different from those of their normal glucose controls (Figure 4(e)).

3.4. Effect of High Glucose on Osteogenic Differentiation of MSCs. To study the effect of high glucose on osteogenic differentiation, BM-MSCs, CH-MSCs, PL-MSCs, and UCMSCs were cultured in osteogenic differentiation medium supplemented with $25 \mathrm{mM}$ D-glucose for 14 days. On culture days 7 and 14, the expression levels of osteogenic genes RUNX2, OSX, and OCN were determined by qRT-PCR. The cultures were also subjected to alkaline phosphatase activity assay on culture day 14 to determine levels of osteogenic differentiation.

Although mRNA levels of RUNX2 and OSX in CHMSCs, PL-MSCs, and UC-MSCs cultured in high glucose were significantly lower than those of their normal glucose controls on culture day 7 (Figure 5(a)), the differences in mRNA levels of those genes could not be detected on culture day 14 (Figure 5(b)). In contrast to RUNX2 and OSX, the mRNA levels of OCN in CH-MSCs, PL-MSCs, and UCMSCs cultured in high glucose condition were not different from those of their normal glucose controls at both time points (Figures 5(a) and 5(b)). In case of BM-MSCs, there was no difference in the mRNA levels of all osteogenic genes examined at both time points, with the exception of $O C N$ in 

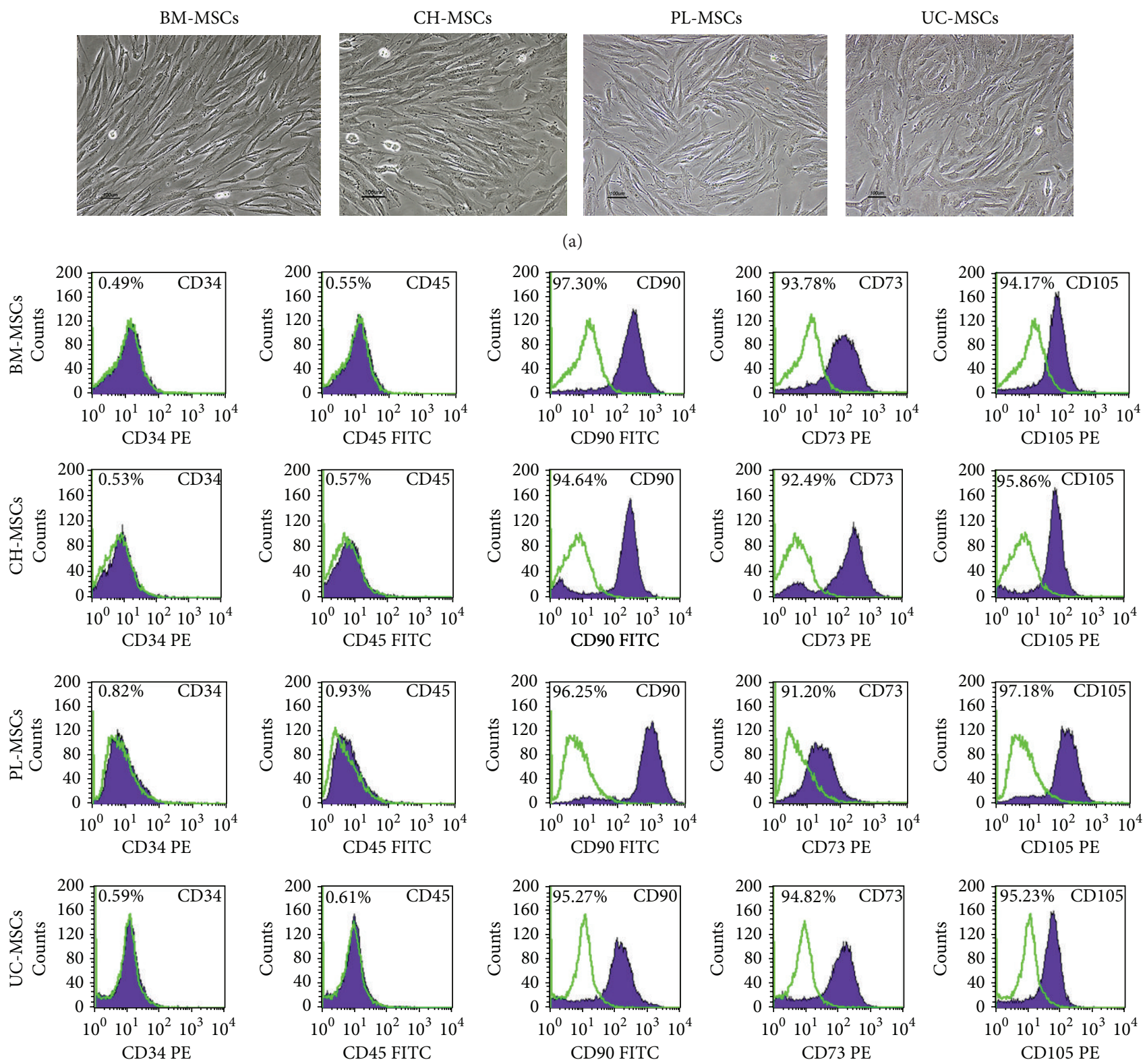

(b)
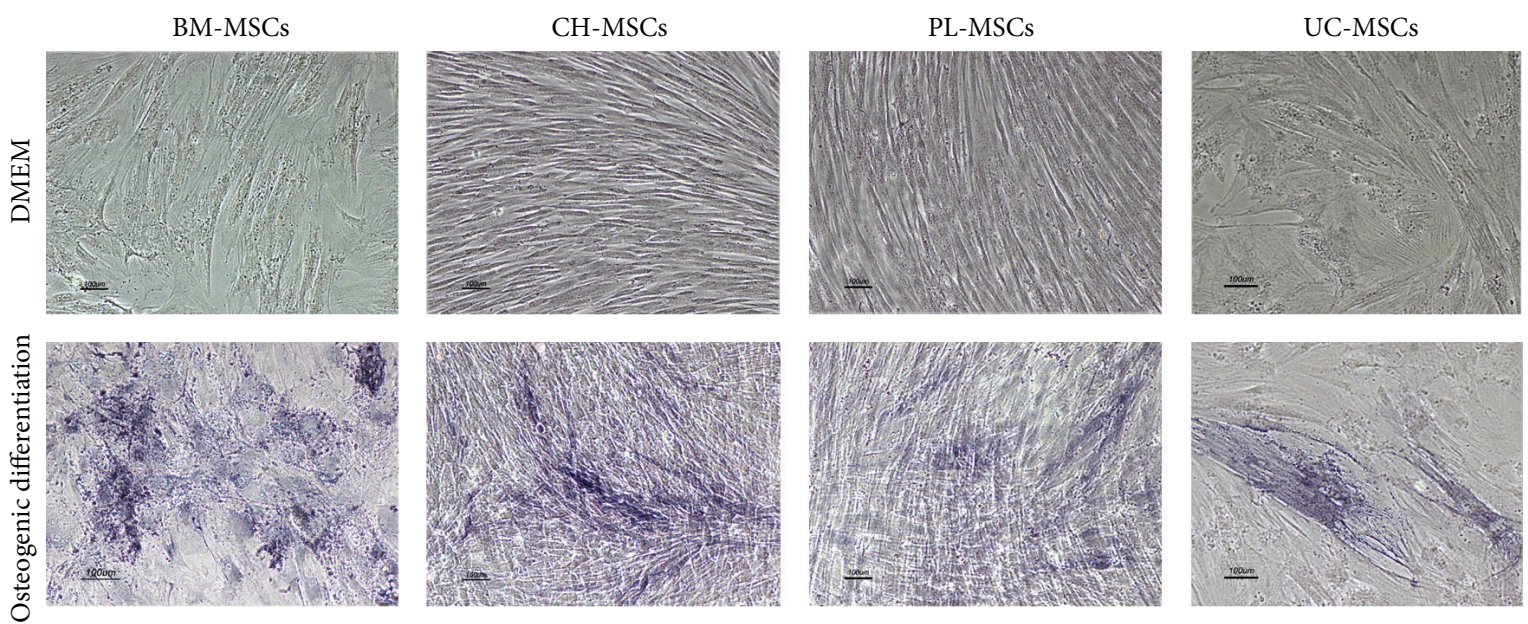

(c)

FIgure 1: Continued. 

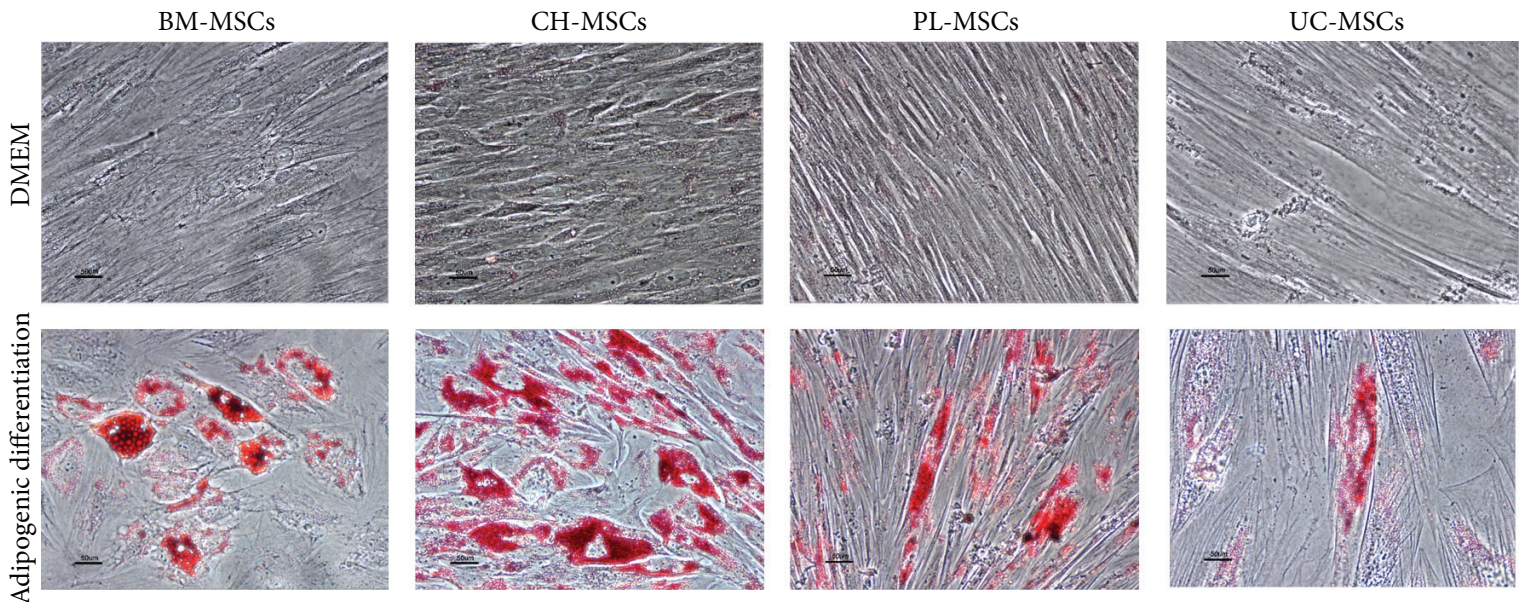

(d)

FIGURE 1: Characteristics of MSCs derived from bone marrow and gestational tissues. (a) Representative micrographs show the fibroblast-like morphology of BM-MSCs, CH-MSCs, PL-MSCs, and UC-MSCs. Scale bar: $100 \mu \mathrm{m}$. (b) Immunophenotypes of BM-MSCs, CH-MSCs, PLMSCs, and UC-MSCs as determined by flow cytometry. (c) Osteogenic differentiation of BM-MSCs, CH-MSCs, PL-MSCs, and UC-MSCs as determined by BCIP/NBT alkaline phosphatase activity assay. Scale bar: $100 \mu \mathrm{m}$. (d) Adipogenic differentiation of BM-MSCs, CH-MSCs, PL-MSCs, and UC-MSCs as determined by oil red O staining. Scale bar: $50 \mu \mathrm{m}$.
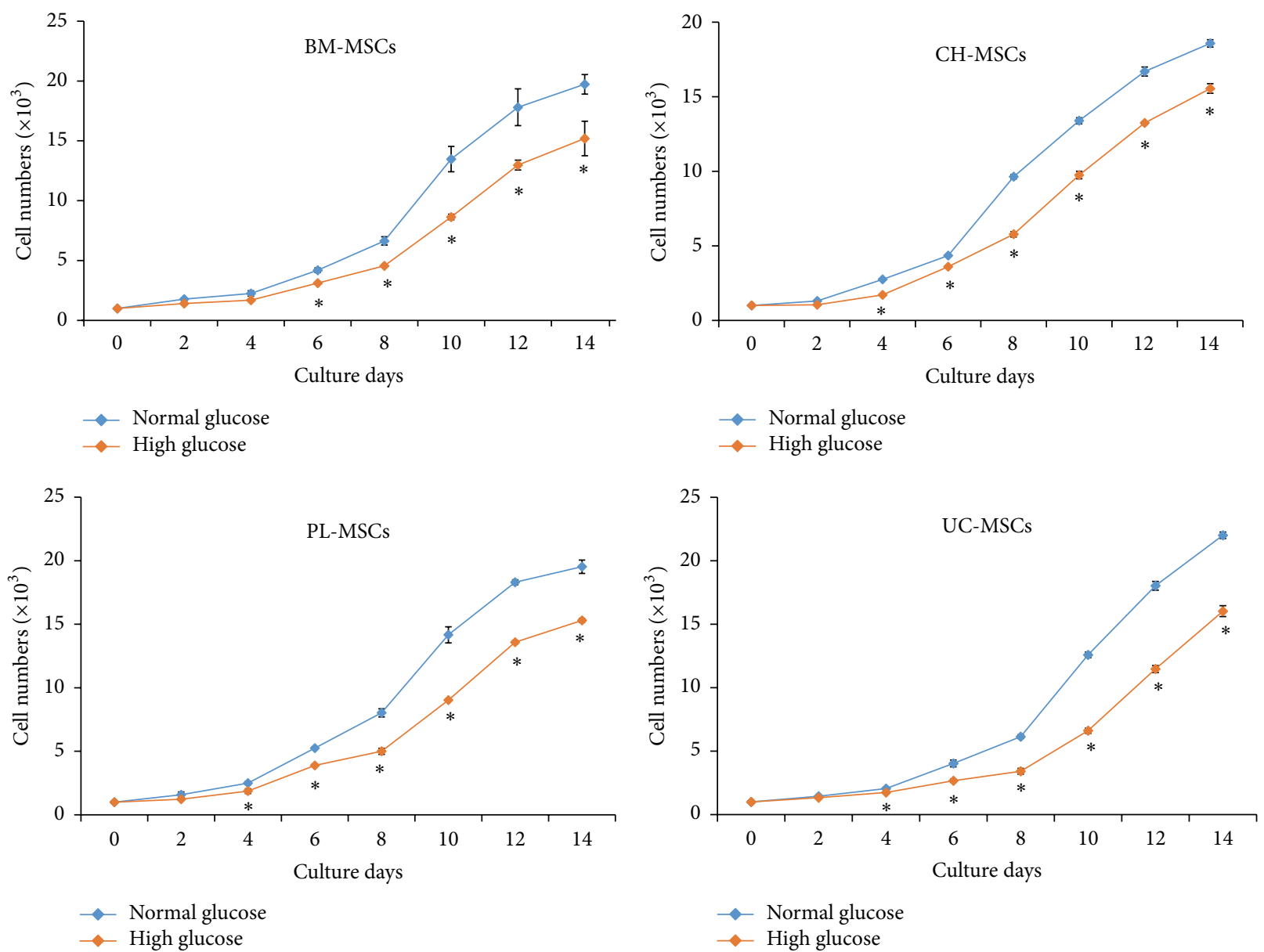

FIGURE 2: Effect of high glucose on MSC proliferation. Figure shows growth kinetic curve of BM-MSCs, CH-MSCs, PL-MSCs, and UCMSCs during a 14-day culture period in high glucose condition (red lines). Data were presented as mean \pm standard error of the mean (SEM) $(n=3) .{ }^{*} P<0.05$ versus normal glucose condition. MSCs cultured in complete medium without glucose supplementation (blue lines) serve as controls. $n$ corresponds to the number of independent samples used in the experiments. 
DMEM

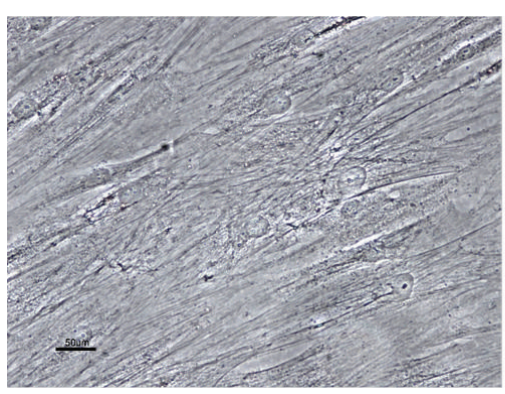

ADIPO

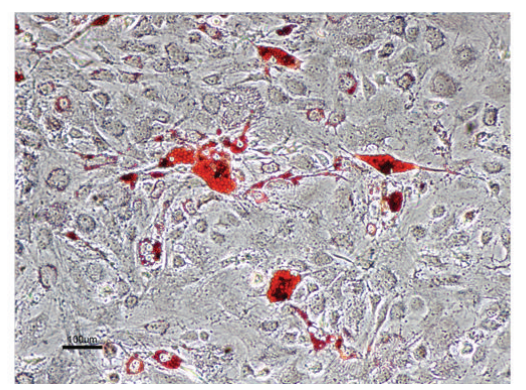

ADIPO + glucose

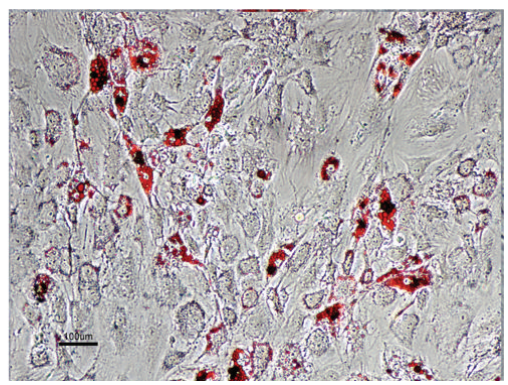

(a)

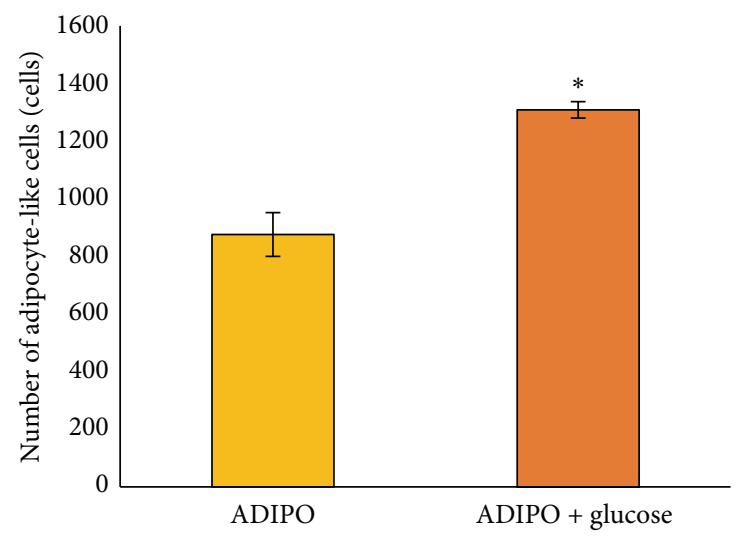

(b)

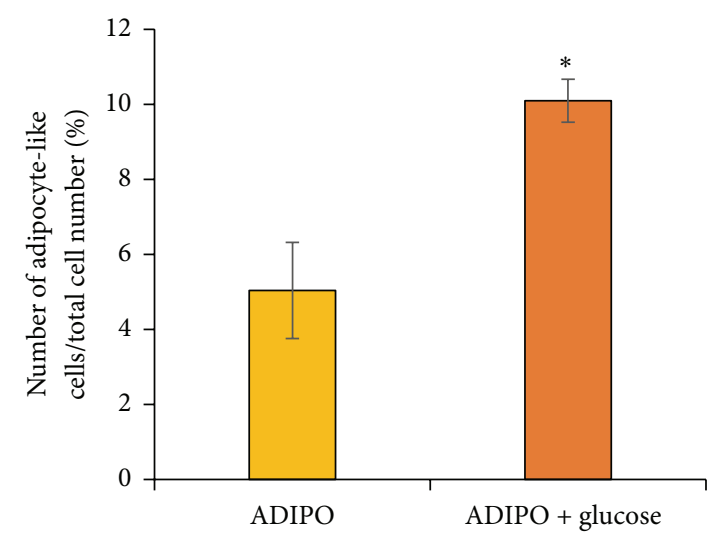

(c)

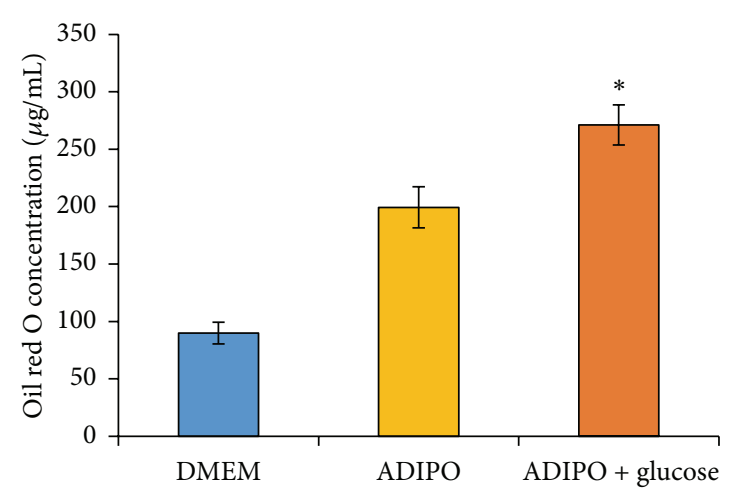

(d)

GLUT4

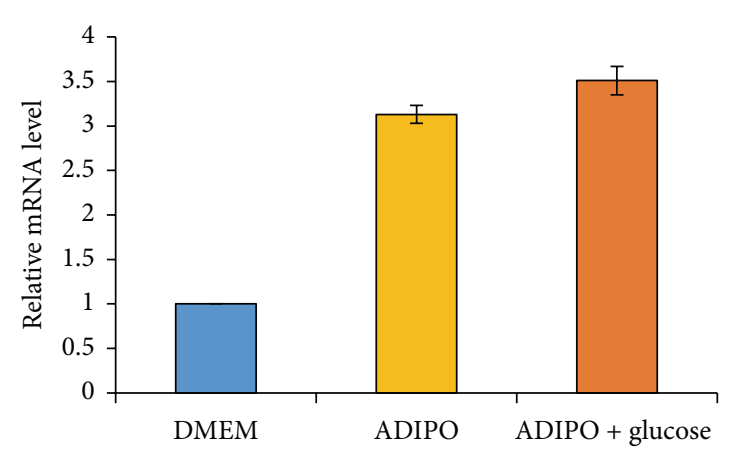

$L P L$

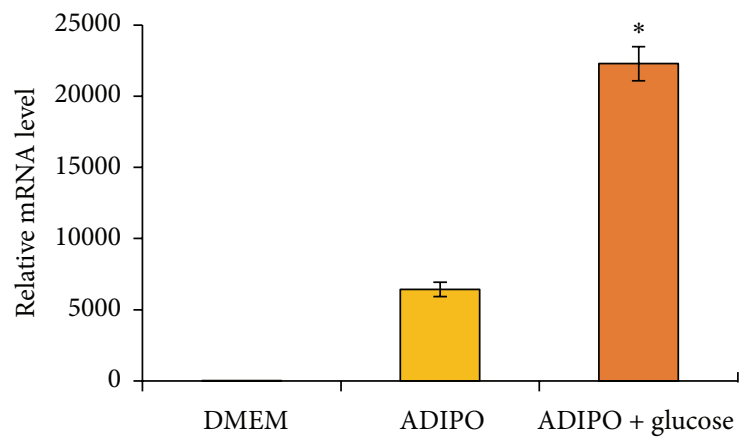

Figure 3: Continued. 

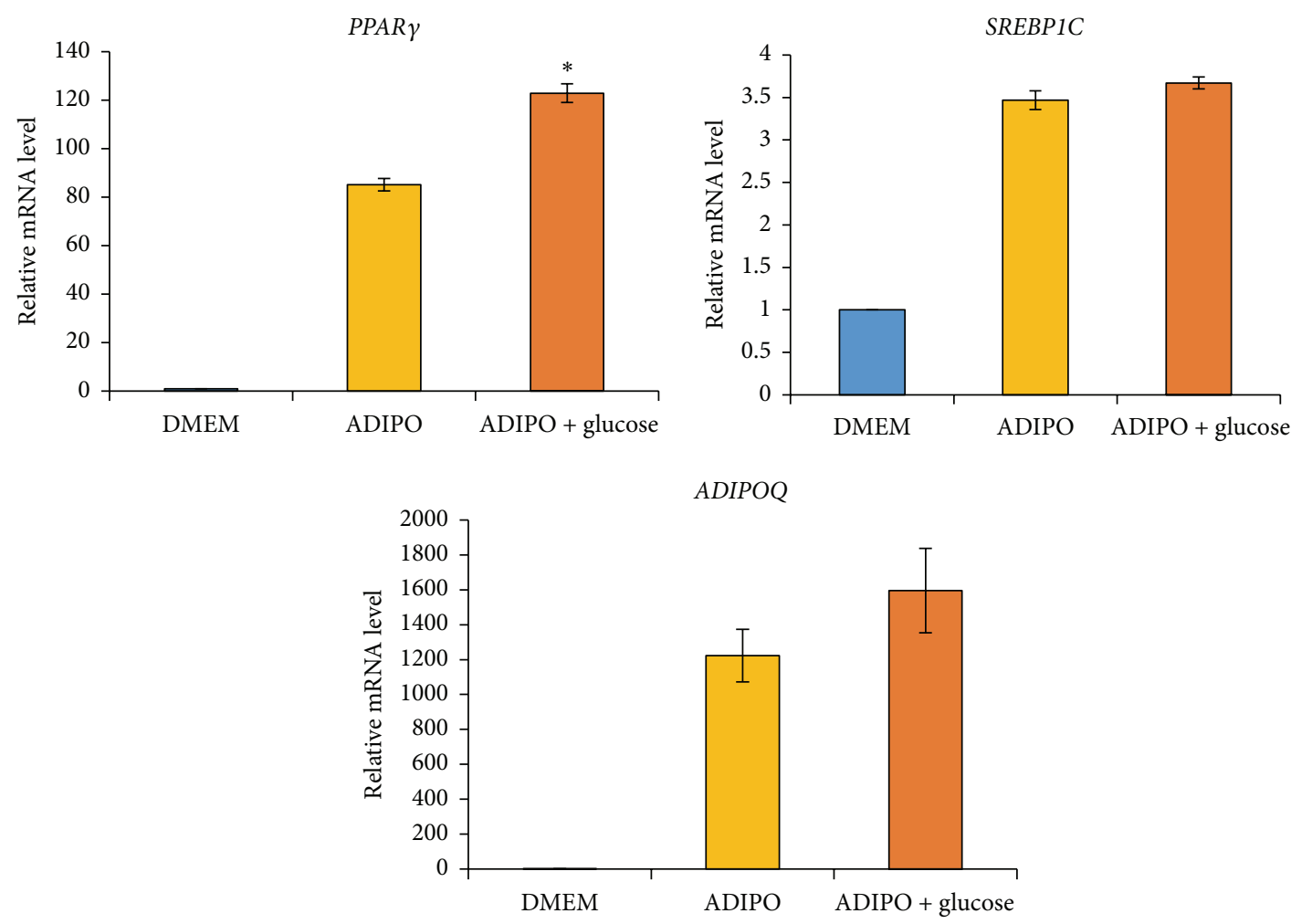

(e)

FIGURE 3: Effect of high glucose on adipogenic differentiation of BM-MSCs. (a) Representative micrographs show the morphology of adipocyte-like cells (red colored cells) derived from BM-MSCs cultured in adipogenic differentiation medium with or without glucose supplementation for 14 days after staining with oil red O. Scale bar: $50 \mu \mathrm{m}$ for DMEM, $100 \mu \mathrm{m}$ for ADIPO, and ADIPO + glucose. (b) Graph shows the number of adipocyte-like cells generated from BM-MSCs cultured in high glucose condition in comparison to their normal glucose controls on culture day $14(n=3)$. (c) Graph shows the percentages of adipocyte-like cells in total cell number generated from BM-MSCs cultured in high glucose condition in comparison to their normal glucose controls on culture day $14(n=3)$. (d) Graph shows the concentration of oil red O staining of BM-MSCs cultured in high glucose condition in comparison to their normal glucose controls on culture day $14(n=3)$. (e) Graph shows relative mRNA levels of adipogenic genes ADIPOQ, GLUT4, LPL, PPAR $\gamma$, and SREBP1C of BM-MSCs cultured in high glucose condition in comparison to their normal glucose controls on culture day $14(n=3)$. Data were presented as mean \pm standard error of the mean (SEM). ${ }^{*} P<0.05$ versus ADIPO. $n$ corresponds to the number of independent samples used in the experiments. DMEM: BM-MSCs cultured in complete medium which serve as nondifferentiation controls. ADIPO: BM-MSCs cultured in adipogenic differentiation medium without glucose supplementation which serve as normal glucose controls. ADIPO + glucose: BM-MSCs cultured in adipogenic differentiation medium supplemented with $25 \mathrm{mM}$ D-glucose.

which its mRNA levels of BM-MSCs cultured in high glucose condition were slightly lower than that of its normal glucose control on culture day 14 (Figure 5(b)).

In agreement with the gene expression study, alkaline phosphatase activity assay showed that the levels of osteogenic differentiation of BM-MSCs, CH-MSCs, PLMSCs, and UC-MSCs in high glucose condition as determined by the alkaline phosphatase activity assay were not obviously different from their normal glucose controls (Figure 5(c)).

\section{Discussion}

Type 2 diabetic patients have increased risks of developing serious long-term complications, such as cardiovascular disease, retinopathy, nephropathy, neuropathy, diabetic foot, and osteoporosis, making diabetes a serious global health problem [1-3]. Most type 2 diabetic patients are obese who have an excessive visceral fat accumulated in their abdominal area. Adipocytes presented in the excessive visceral fat release several factors including fatty acids, glycerol, hormones, and proinflammatory cytokines that increase insulin resistance making diabetic treatment ineffective [12].

It is known that adipocytes, which are the major components of fat tissues, are derived, at least in part, from mesenchymal stem cells [13]. Although the effects of high glucose on the proliferation and adipogenic and osteogenic differentiation of mouse and rat BM-MSCs have been previously reported $[6,8,9]$, the effects of high glucose on the properties of gestational tissue-derived MSCs especially on their adipogenic and osteogenic differentiation are currently unknown.

In the present study, we successfully isolated MSCs from several gestational tissues including CH-MSCs, PL-MSCs, 

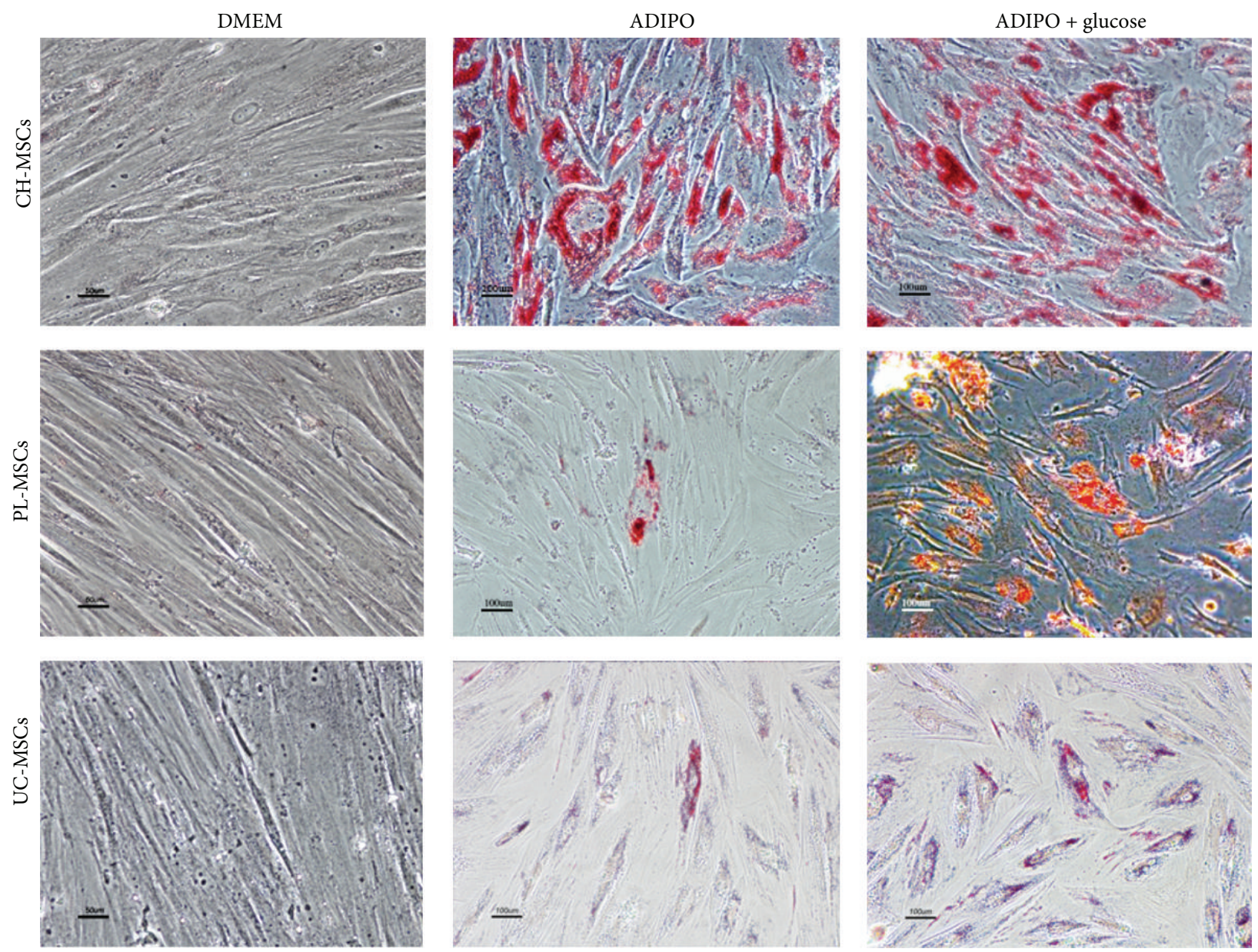

(a)
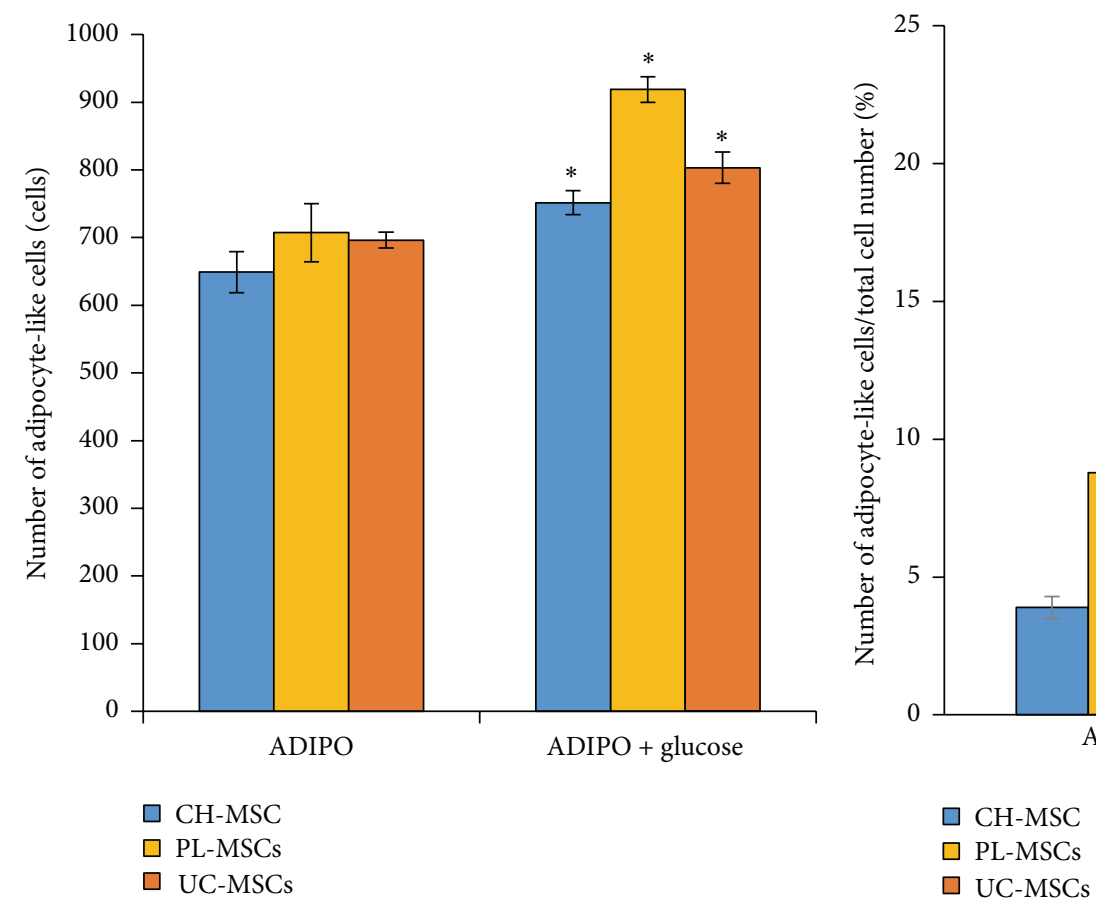

(b)

(c)

Figure 4: Continued. 


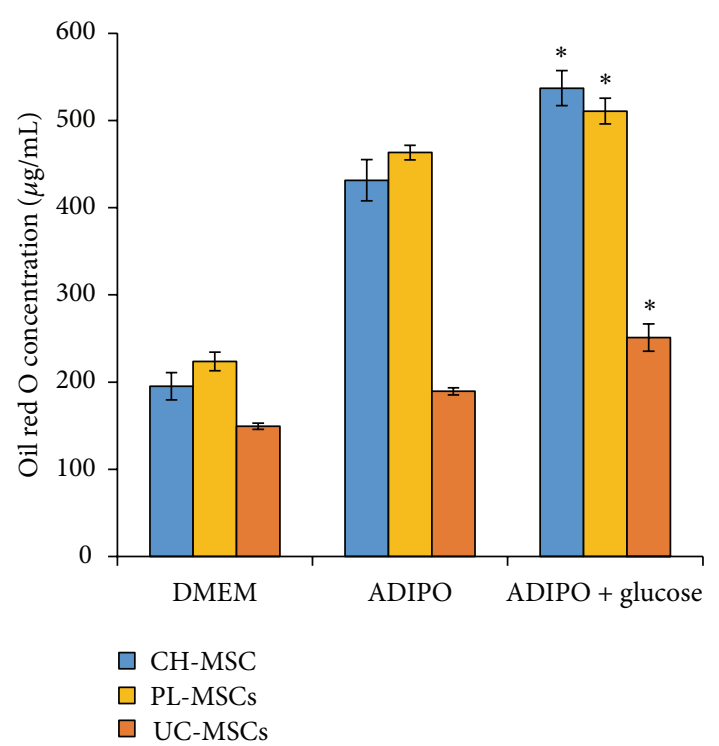

(d)

GLUT4
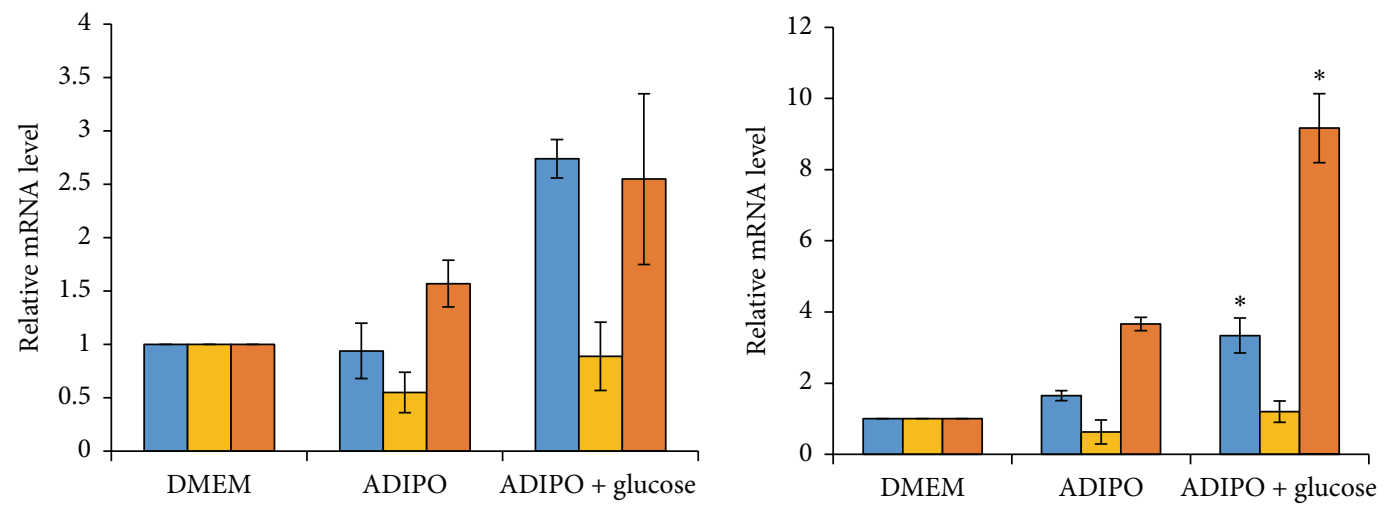

$\square \mathrm{CH}-\mathrm{MSCs}$

CH-MSCs

$\square$ PL-MSCs

$\square$ PL-MSCs

$\square$ UC-MSCs

$\checkmark$ UC-MSCs
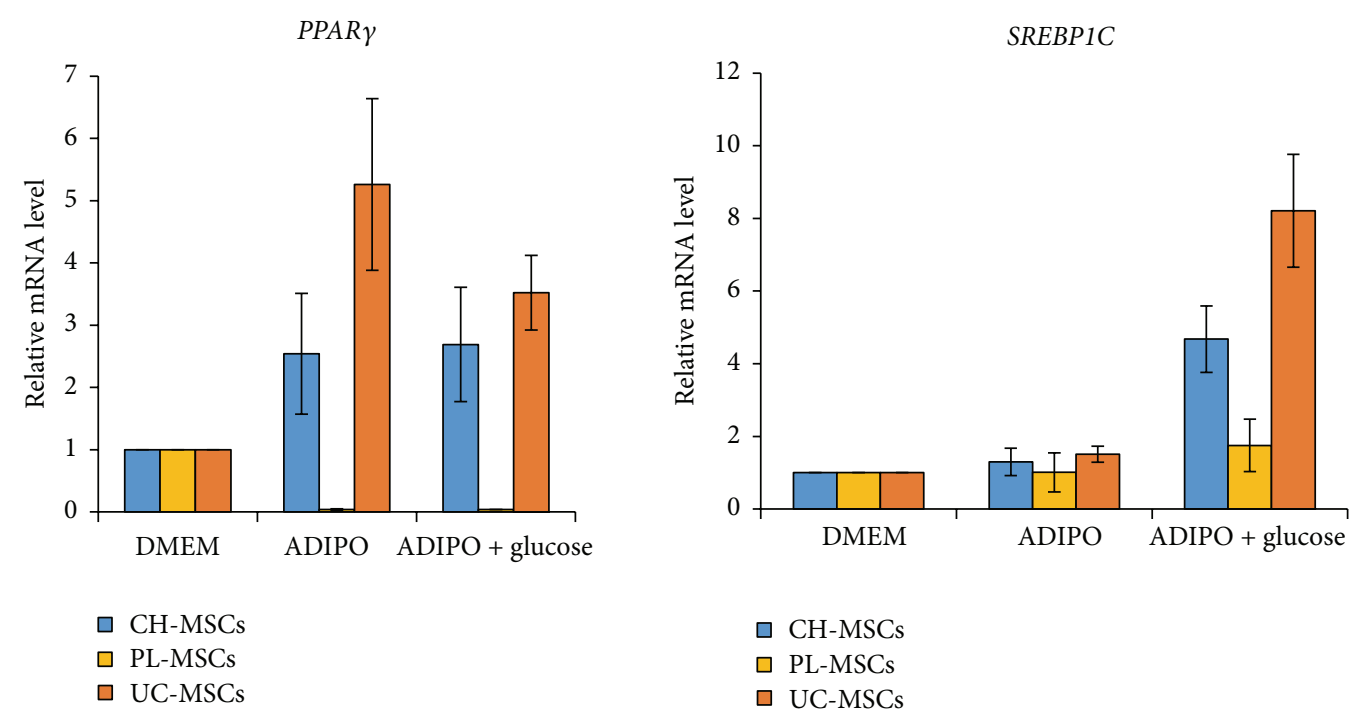

FIgUre 4: Continued. 


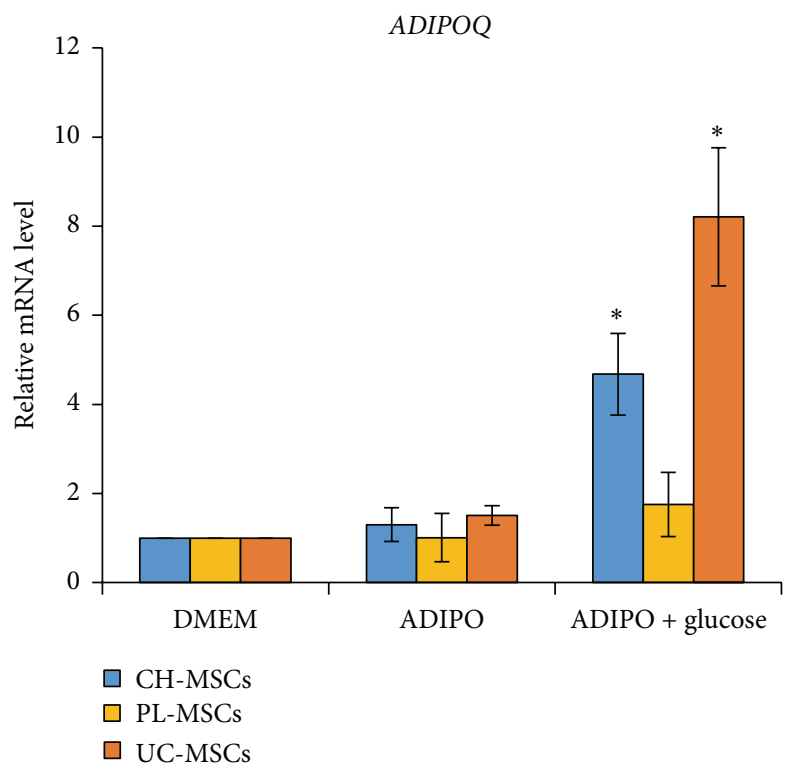

(e)

FIGURE 4: Effect of high glucose on adipogenic differentiation of gestational tissue-derived MSCs. (a) Representative micrographs show the morphology of adipocyte-like cells (red colored cells) derived from CH-MSCs, PL-MSCs, and UC-MSCs cultured in adipogenic differentiation medium with or without glucose supplementation for 28 days after staining with oil red O. Scale bar: $50 \mu \mathrm{m}$ for DMEM, $100 \mu \mathrm{m}$ for ADIPO, and ADIPO + glucose. (b) Graph shows the number of adipocyte-like cells generated from CH-MSCs, PL-MSCs, and UC-MSCs cultured in high glucose condition in comparison to their normal glucose controls on culture day $28(n=3)$. (c) Graph shows the percentages of adipocyte-like cells in total cell number generated from CH-MSCs, PL-MSCs, and UC-MSCs cultured in high glucose condition in comparison to their normal glucose controls on culture day $28(n=3)$. (d) Graph shows the concentrations of oil red O staining of CH-MSCs, PL-MSCs, and UC-MSCs cultured in high glucose condition in comparison to their normal glucose controls on culture day 28 $(n=3)$. (e) Graph shows relative mRNA levels of adipogenic genes ADIPOQ, GLUT4, LPL, PPAR , and SREBP1C of CH-MSCs, PL-MSCs, and UC-MSCs cultured in high glucose condition in comparison to their normal glucose controls on culture day $28(n=3)$. Data were presented as mean \pm standard error of the mean (SEM). ${ }^{*} P<0.05$ versus ADIPO. $n$ corresponds to the number of independent samples used in the experiments. DMEM: MSCs cultured in complete medium which serve as nondifferentiation controls. ADIPO: MSCs cultured in adipogenic differentiation medium without glucose supplementation which serve as normal glucose controls. ADIPO + glucose: MSCs cultured in adipogenic differentiation medium supplemented with $25 \mathrm{mM} \mathrm{D}$-glucose.

and UC-MSCs. Similar to BM-MSCs, these gestational tissuederived MSCs displayed fibroblast-like morphology, exhibited typical MSC surface markers, and could differentiate to osteocytes and adipocytes. The characteristics of $\mathrm{CH}$ MSCs, PL-MSCs, and UC-MSCs established in this study were similar to those described in the previous reports [14-19]. Our observation showed that CH-MSCs, PL-MSCs, and UC-MSCs took a longer period of time than BMMSCs to differentiate to adipocytes and osteocytes and the numbers of adipocytes and osteocytes derived from these MSC sources were lower than those derived from BM-MSCs cultured under the same conditions. These results reflect the endogenous differences in the osteogenic and adipogenic differentiation capacity between gestational tissue-derived MSCs and BM-MSCs.

Similar to BM-MSCs, high glucose suppressed the proliferation of CH-MSCs, PL-MSCs, and UC-MSCs. This result is in agreement with previous reports showing that high glucose inhibited proliferation of mouse BM-MSCs $[7,8]$ and human osteosarcoma MG63 cells $[9,10]$ by modulating JAK/STAT and p38 signaling pathways $[20,21]$.
Although previous studies demonstrated that high glucose enhanced adipogenic differentiation of mouse BMMSCs [7, 8], MG63 cell [9], and human muscle-derived stem cells [11] by upregulating the expression of several adipogenic genes $[8,9,11]$, the effects of high glucose on adipogenic differentiation of gestational tissue-derived MSCs have not yet been determined. This study demonstrates for the first time that high glucose induced the expression of adipogenic gene PPAR $\gamma$ and LPL in BM-MSCs, as well as ADIPOQ and LPL in CH-MSCs, PL-MSCs, and UC-MSCs. The upregulation of these key regulators of adipogenesis enhanced adipogenic differentiation of BM-MSCs, CH-MSCs, PL-MSCs, and UCMSCs as demonstrated by an increase of both adipocyte number and extent of oil red O staining in MSCs cultured in high glucose condition. These results are in agreement with the previous studies which reported that high glucose upregulated the expression of several adipogenic genes including PPAR $\gamma$, SREBPIC, ADIPOQ, LPL, and GLUT4 in mouse BMMSCs, MG63 cells and human muscle-derived stem cells $[8,9,11]$. 

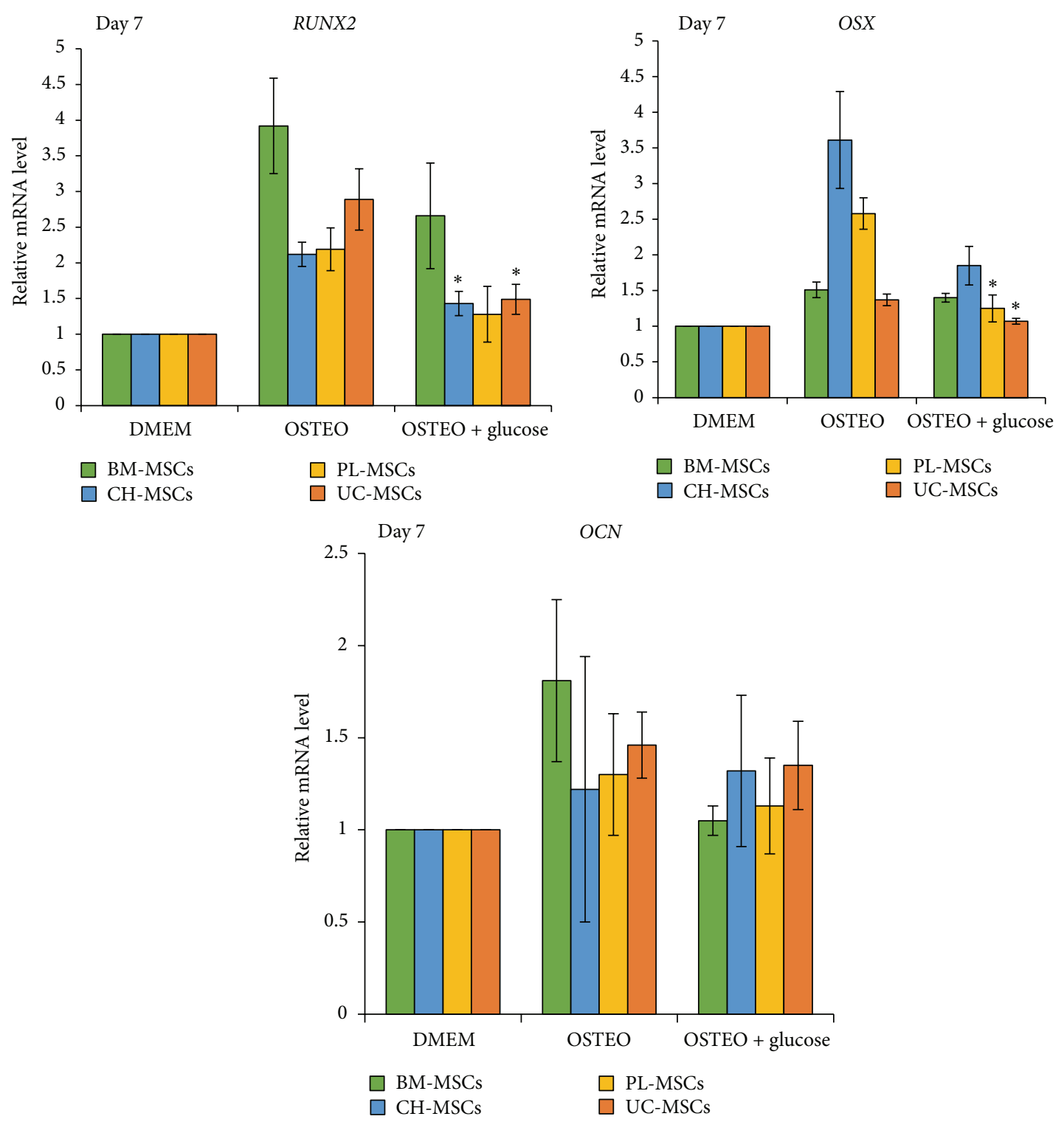

(a)
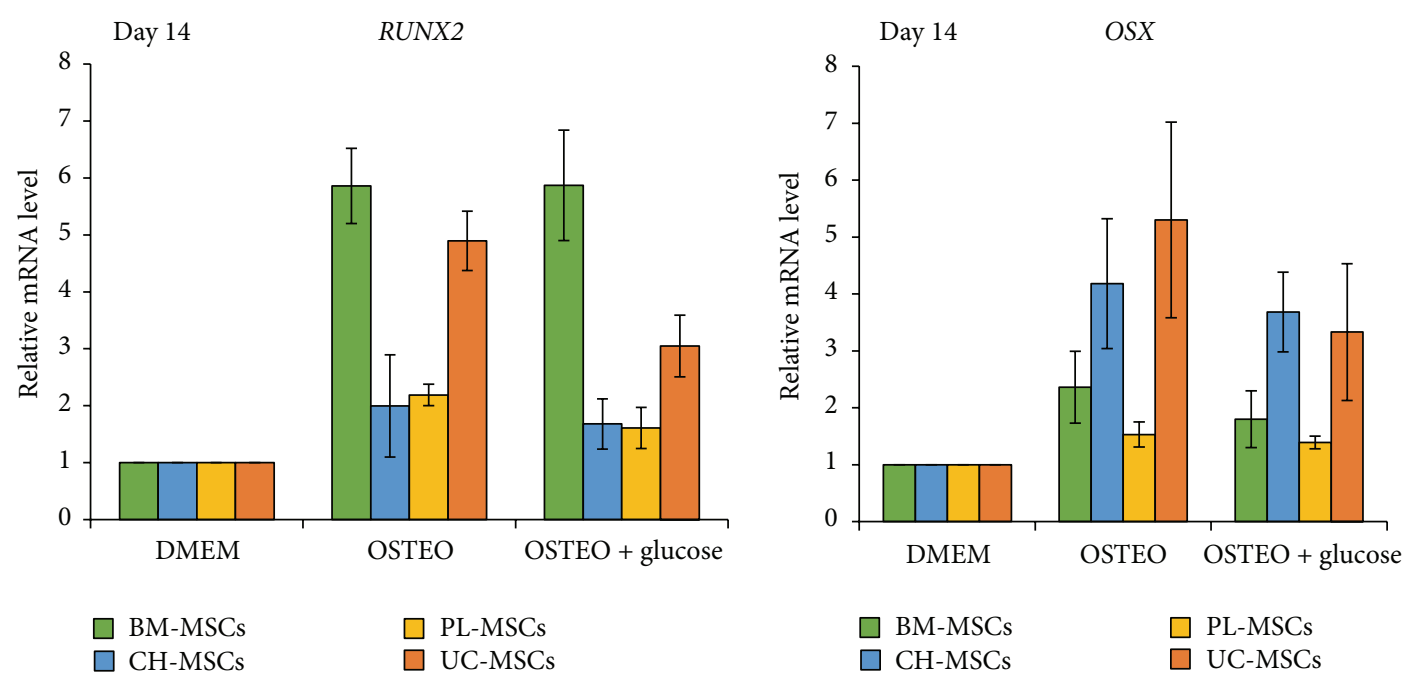

Figure 5: Continued. 


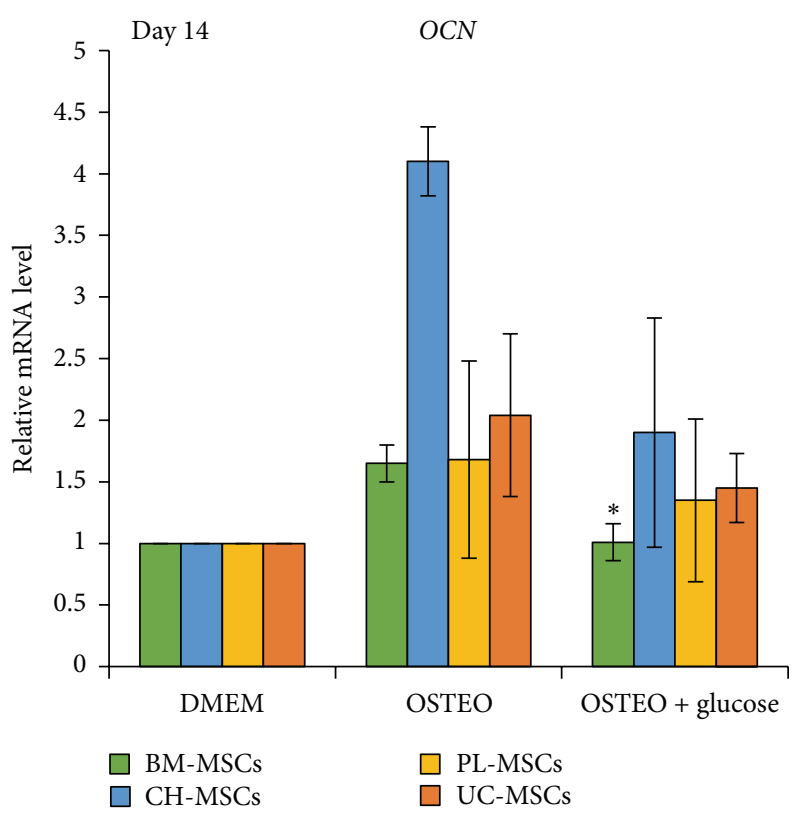

(b)
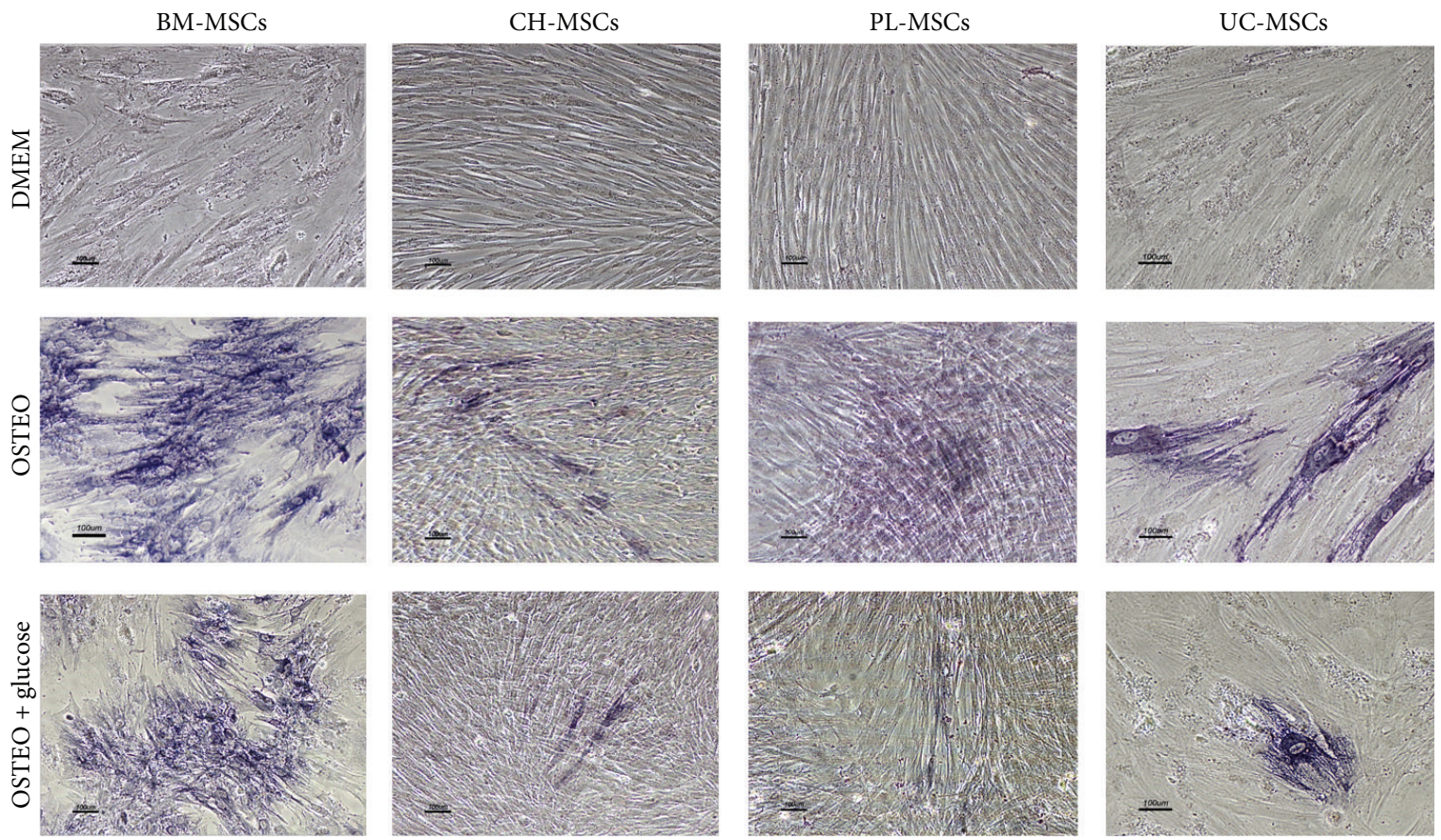

(c)

FIGURE 5: The effect of high glucose on osteogenic differentiation of BM-MSCs, CH-MSCs, PL-MSCs, and UC-MSCs. (a) Graph shows relative mRNA levels of osteogenic genes RUNX2, OSX, and OCN of BM-MSCs, CH-MSCs, PL-MSCs, and UC-MSCs cultured in high glucose condition in comparison to their normal glucose controls on culture day $7(n=3)$. (b) Graph shows relative mRNA levels of osteogenic genes RUNX2, OSX, and OCN of BM-MSCs, CH-MSCs, PL-MSCs, and UC-MSCs cultured in high glucose condition in comparison to their normal glucose controls on culture day $14(n=3)$. (c) Representative micrographs show the morphology of osteocyte-like cells (purple colored cells) derived from BM-MSCs, CH-MSCs, PL-MSCs, and UC-MSCs cultured in osteogenic differentiation medium with or without glucose supplementation for 14 days after being subjected to BCIP/NBT alkaline phosphatase activity assay. Scale bar: $100 \mu \mathrm{m}$. Data were presented as mean \pm standard error of the mean (SEM). ${ }^{*} P<0.05$ versus OSTEO. $n$ corresponds to the number of independent samples used in the experiments. DMEM: MSCs cultured in complete medium which serve as nondifferentiation controls. OSTEO: MSCs cultured in osteogenic differentiation medium without glucose supplementation which serve as normal glucose controls. OSTEO + glucose: MSCs cultured in osteogenic differentiation medium supplemented with $25 \mathrm{mM}$ D-glucose. 
We observed both similarity and variability in adipogenic gene expression between bone marrow- and gestational tissue-derived MSCs which were cultured in high glucose condition. While high glucose clearly upregulated the expression of LPL gene in both bone marrow- and gestational tissuederived MSCs, its effects on ADIPOQ and PPAR $\gamma$ gene seem to be tissue specific. High glucose upregulated the expression of ADIPOQ only in CH-MSCs and UC-MSCs but not in BMMSCs. In contrast, high glucose upregulated the expression of PPAR $\gamma$ only in BM-MSCs but not in gestational tissuederived MSCs.

The differences in adipogenic gene expression might arise from two possibilities. Firstly, there are endogenous differences in the adipogenic differentiation capacity between bone marrow- and gestational tissue-derived MSCs. Our results showed that the upregulation of ADIPOQ and PPAR $\gamma$ genes in BM-MSCs cultured in adipogenic differentiation medium was much greater than those of gestational tissue-derived MSCs cultured under the same condition. These results suggest that BM-MSCs have higher adipogenic differentiation capacity than gestational tissue-derived MSCs. The upregulation of $A D I P O Q$ gene in BM-MSCs cultured in adipogenic differentiation medium which is already 1200 -fold higher than control even without glucose supplementation might obscure the positive effect of high glucose on the expression of this gene. In contrast to BM-MSCs, there is no upregulation of ADIPOQ gene in CH-MSCs and UC-MSCs cultured in adipogenic differentiation medium without glucose supplementation, so that the positive effect of high glucose on the expression of this gene became apparent. Secondly, it might also be possible that the adipocytes derived from gestational tissuederived MSCs might be less mature than those derived from BM-MSCs; therefore, the expression levels of several adipogenic genes, such as $A D I P O Q, P P A R \gamma$, and $L P L$, were much lower than those of BM-MSCs. This was supported by our observation that the adipocytes generated from gestational tissue-derived MSCs contain less amount of lipid droplets in comparison to the adipocytes generated from BM-MSCs.

High glucose might induce adipogenic differentiation of bone marrow- and gestational tissue-derived MSCs by increasing the production of reactive oxygen species (ROS) [11]. The increasing level of ROS has been reported to induce PKC signalling and lead to the adipogenic differentiation of both human adipose- and skeletal muscle-derived stem cells [11].

With regard to the osteogenic differentiation, we found that high glucose downregulated the expression of osteogenic gene RUNX2 in CH-MSCs and UC-MSCs as well as OSX in CH-MSCs, PL-MSCs, and UC-MSCs on culture day 7. However, the downregulation of those genes could not be detected on culture day 14 . We also did not find any difference in the levels of osteogenic differentiation of BM-MSCs, $\mathrm{CH}$ MSCs, PL-MSCs, and UC-MSCs cultured under high glucose condition in comparison to their normal glucose controls.

Our results suggest that the effect of high glucose on the expression of osteogenic genes in MSCs is transient and its effect on the osteogenic differentiation of both BMMSCs and gestational tissue-derived MSCs was not clearly demonstrated. These results are in contrast to the previous studies showing that high glucose suppressed the expression of osteogenic genes RUNX2, OCN, COL1A1, and ON in MG63 cells by activating cAMP/PKA/ERK pathway $[9,10]$. This contrary is most likely arising from differences in biological properties between MG63 and gestational tissue-derived MSCs and the way they respond to high glucose.

\section{Conclusion}

We herein reported for the first time the effects of high glucose on the proliferation and adipogenic and osteogenic differentiation of several gestational tissue-derived MSCs including CH-MSCs, PL-MSCs, and UC-MSCs. Our result demonstrated that high glucose inhibits proliferation and enhances adipogenic differentiation of BM-MSCs, $\mathrm{CH}$ MSCs, PL-MSCs, and UC-MSCs by upregulating the expressions of adipogenic genes $P P A R \gamma, A D I P O Q$, and $L P L$. In contrast to adipogenic differentiation, high glucose did not affect osteogenic differentiation of all MSCs examined in this study. The knowledge gained from this study will increase our understanding on the mechanisms underlying the effects of high glucose on proliferation and adipogenic and osteogenic differentiation of MSCs and might lead to an improvement in the treatments of diabetes, obesity, and metabolic syndrome in the near future.

\section{Disclosure}

There were no commercial organizations or funding bodies associated with data collection and analysis or this paper.

\section{Conflict of Interests}

The authors declare no conflict of interests.

\section{Authors' Contribution}

Weerawan Hankamolsiri performed the experiments; Sirikul Manochantr, Chairat Tantrawatpan, Duangrat Tantikanlayaporn, and Pairath Tapanadechopone analyzed the data; Pakpoom Kheolamai conceived, designed, and supervised the study, analyzed the data, and wrote the paper. All authors read and approved the final paper.

\section{Acknowledgment}

This research project was funded by grant from Thammasat University.

\section{References}

[1] M. Brownlee, "The pathobiology of diabetic complications: a unifying mechanism," Diabetes, vol. 54, no. 6, pp. 1615-1625, 2005.

[2] D. M. Nathan, P. A. Cleary, J.-Y. C. Backlund et al., "Intensive diabetes treatment and cardiovascular disease in patients with type 1 diabetes," The New England Journal of Medicine, vol. 353, no. 25, pp. 2643-2653, 2005. 
[3] S. Wild, G. Roglic, A. Green, R. Sicree, and H. King, "Global prevalence of diabetes: estimates for the year 2000 and projections for 2030," Diabetes Care, vol. 27, no. 5, pp. 1047-1053, 2004.

[4] N. Freemantle, J. Holmes, A. Hockey, and S. Kumar, "How strong is the association between abdominal obesity and the incidence of type 2 diabetes?" International Journal of Clinical Practice, vol. 62, no. 9, pp. 1391-1396, 2008.

[5] J.-P. Després and I. Lemieux, "Abdominal obesity and metabolic syndrome," Nature, vol. 444, no. 7121, pp. 881-887, 2006.

[6] A. Stolzing, N. Coleman, and A. Scutt, "Glucose-induced replicative senescence in mesenchymal stem cells," Rejuvenation Research, vol. 9, no. 1, pp. 31-35, 2006.

[7] J. Wang, W. Bin, L. Ying et al., "High glucose inhibits osteogenic differentiation through the BMP signaling pathway in bone mesenchymal stem cells in mice," EXCLI Journal, vol. 12, pp. 584-597, 2014.

[8] C. C. Chia, S. Y. Rong, S. T. Keh, M. H. Feng, and S. H. Liu, "Hyperglycemia enhances adipogenic induction of lipid accumulation: involvement of extracellular signal-regulated protein kinase 1/2, phosphoinositide 3-kinase/Akt, and peroxisome proliferator-activated receptor gamma signaling," Endocrinology, vol. 148, no. 9, pp. 4267-4275, 2007.

[9] W. Wang, X. Zhang, J. Zheng, and J. Yang, "High glucose stimulates adipogenic and inhibits osteogenic differentiation in MG63 cells through cAMP/protein kinase A/extracellular signalregulated kinase pathway," Molecular and Cellular Biochemistry, vol. 338, no. 1-2, pp. 115-122, 2010.

[10] X. Shao, X. Cao, G. Song, Y. Zhao, and B. Shi, "Metformin rescues the MG63 osteoblasts against the effect of high glucose on proliferation," Journal of Diabetes Research, vol. 2014, Article ID 453940, 6 pages, 2014.

[11] P. Aguiari, S. Leo, B. Zavan et al., "High glucose induces adipogenic differentiation of muscle-derived stem cells," Proceedings of the National Academy of Sciences of the United States of America, vol. 105, no. 4, pp. 1226-1231, 2008.

[12] S. E. Kahn, B. Zinman, S. M. Haffner et al., "Obesity is a major determinant of the association of C-reactive protein levels and the metabolic syndrome in type 2 diabetes," Diabetes, vol. 55, no. 8, pp. 2357-2364, 2006.

[13] J. Moreno-Navarrete and J. Fernández-Real, "Adipocyte differentiation," in Adipose Tissue Biology, M. E. Symonds, Ed., pp. 17-38, Springer, New York, NY, USA, 2012.

[14] D. L. Mackay, P. J. Tesar, L.-N. Liang, and S. E. Haynesworth, "Characterizing medullary and human mesenchymal stem cellderived adipocytes," Journal of Cellular Physiology, vol. 207, no. 3, pp. 722-728, 2006.

[15] D. J. Klemm, J. W. Leitner, P. Watson et al., "Insulin-induced adipocyte differentiation: activation of CREB rescues adipogenesis from the arrest caused by inhibition of prenylation," Journal of Biological Chemistry, vol. 276, no. 30, pp. 28430-28435, 2001.

[16] J. E. Dennis and P. Charbord, "Origin and differentiation of human and murine stroma," Stem Cells, vol. 20, no. 3, pp. 205$214,2002$.

[17] M. F. Pittenger, A. M. Mackay, S. C. Beck et al., "Multilineage potential of adult human mesenchymal stem cells," Science, vol. 284, no. 5411, pp. 143-147, 1999.

[18] M. Dominici, K. Le Blanc, I. Mueller et al., "Minimal criteria for defining multipotent mesenchymal stromal cells. The International Society for Cellular Therapy position statement," Cytotherapy, vol. 8, no. 4, pp. 315-317, 2006.
[19] E. M. Horwitz, K. Le Blanc, M. Dominici et al., "Clarification of the nomenclature for MSC: the International Society for Cellular Therapy position statement," Cytotherapy, vol. 7, no. 5, pp. 393-395, 2005.

[20] Z. Liu, M. Lei, Y. Jiang et al., "High glucose attenuates VEGF expression in rat multipotent adult progenitor cells in association with inhibition of JAK2/STAT3 signalling," Journal of Cellular and Molecular Medicine, vol. 13, no. 9, pp. 3427-3436, 2009.

[21] S. Kuki, T. Imanishi, K. Kobayashi, Y. Matsuo, M. Obana, and T. Akasaka, "Hyperglycemia accelerated endothelial progenitor cell senescence via the activation of p38 mitogen-activated protein kinase," Circulation Journal, vol. 70, no. 8, pp. 1076-1081, 2006. 

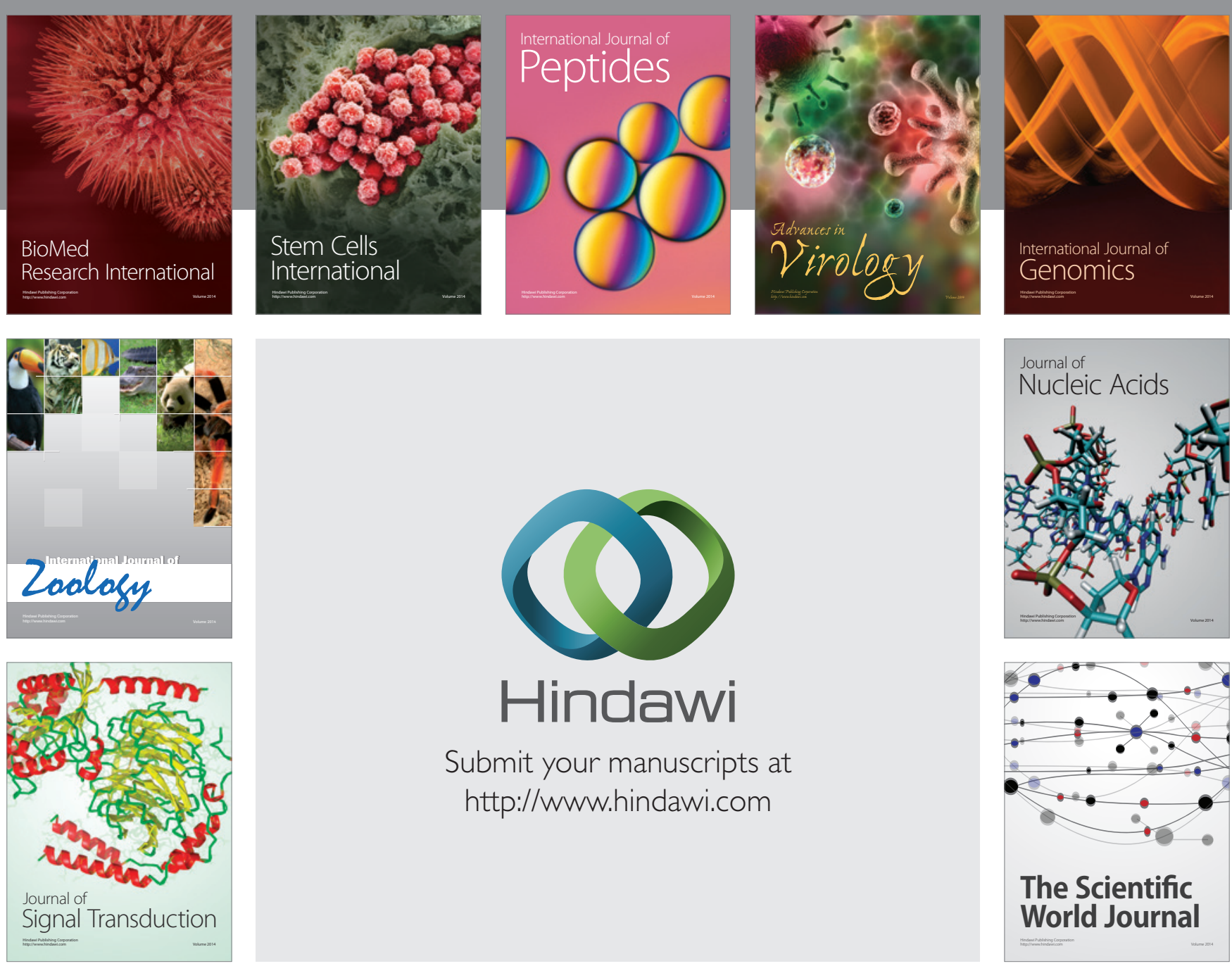

Submit your manuscripts at

http://www.hindawi.com
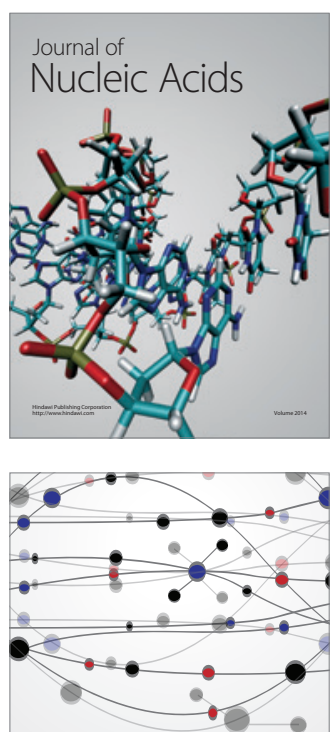

The Scientific World Journal
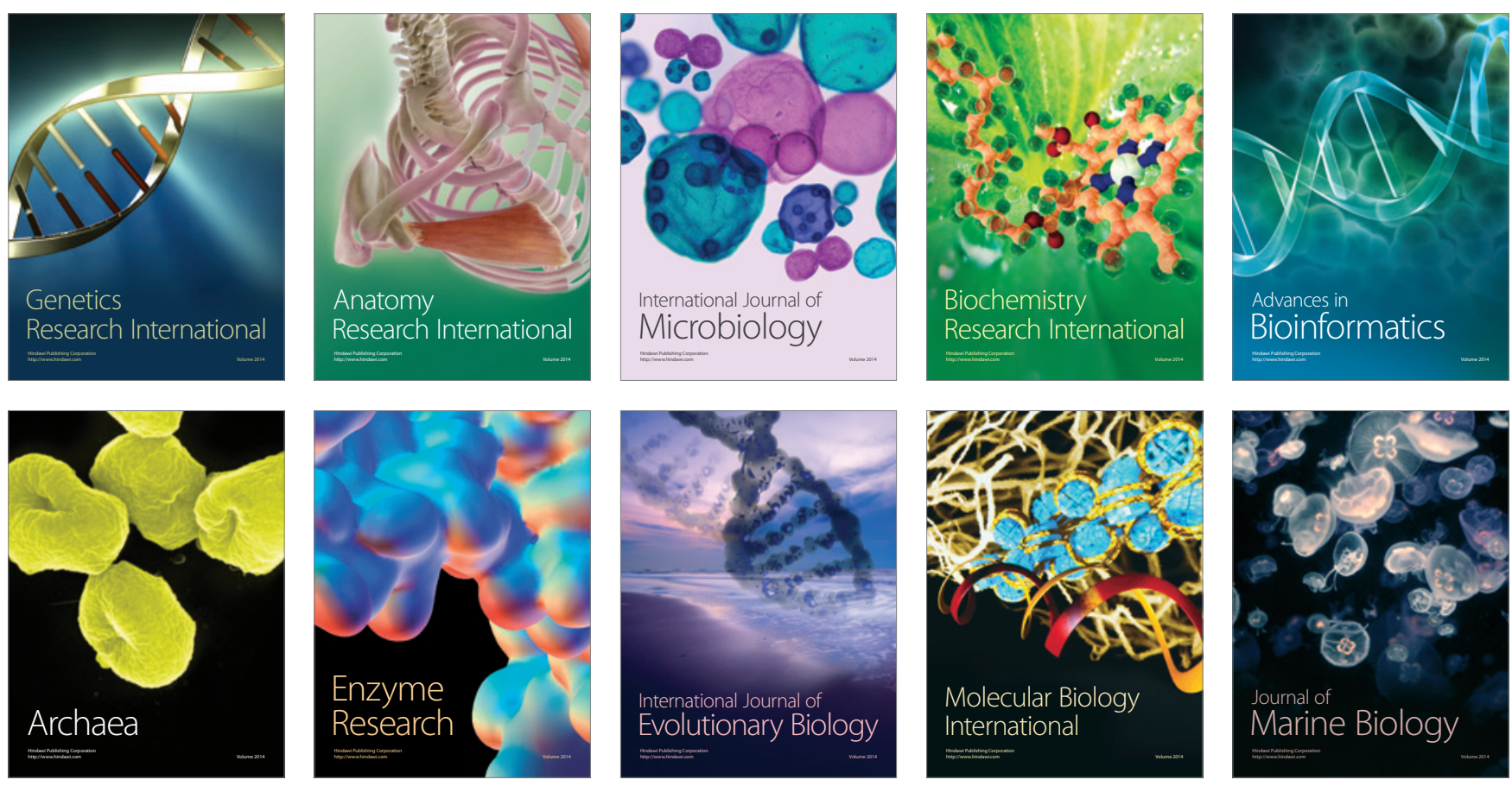